\title{
Use of Non-canonical Tyrosine Analogs to Probe Control of Radical Intermediates during Endoperoxide Installation by Verruculogen Synthase (FtmOx1)
}

Chi-Yun Lin, ${ }^{*}, 1$ Angel L. Muñoz, ${ }^{2}$ Tatiana N. Laremore, ${ }^{3}$ Alexey Silakov, $,{ }^{*}, 1$ Carsten

Krebs, , ${ }^{*}, 1,2$ Amie K. Boal, ${ }^{*}, 1,2$ J. Martin Bollinger, Jr. ${ }^{*}, 1,2$

${ }^{1}$ Department of Chemistry, The Pennsylvania State University; University Park, PA 16802, USA.

2Department of Biochemistry and Molecular Biology, The Pennsylvania State University; University Park, PA 16802, USA.

${ }^{3}$ Huck Institutes of the Life Sciences, The Pennsylvania State University; University Park, PA 16802, USA.

*Corresponding author. Email: chiyun1521@gmail.com; aus40@psu.edu;

ckrebs@psu.edu; akb20@psu.edu; jmb21@psu.edu 


\section{ABSTRACT}

Important bioactive natural products, including prostaglandin $\mathrm{H}_{2}$ and artemisinin, contain reactive endoperoxides. Known enzymatic pathways for endoperoxide installation require multiple hydrogen-atom transfers (HATs). For example, iron(II)- and 2oxoglutarate-dependent verruculogen synthase (FtmOx1; EC 1.14.11.38) mediates HAT from aliphatic $\mathrm{C} 21$ of fumitremorgin $\mathrm{B}$, capture of $\mathrm{O}_{2}$ by the $\mathrm{C} 21$ radical $(\mathrm{C} 21 \cdot)$, addition of the peroxyl radical (C21-O-O•) to olefinic $\mathrm{C} 27$, and HAT to the resultant $\mathrm{C} 26 \cdot$. Recent studies proposed conflicting roles for FtmOx1 tyrosine residues, Tyr224 and Tyr68, in the HATs from $\mathrm{C} 21$ and to $\mathrm{C} 26 \cdot$. Here, analysis of variant proteins bearing a ringhalogenated tyrosine or (amino)phenylalanine in place of either residue establishes that Tyr68 is the hydrogen donor to $\mathrm{C} 26 \cdot$, while Tyr224 has no essential role. The radicals that accumulate rapidly in FtmOx1 variants bearing a HAT-competent tyrosine analog at position 68 exhibit hypsochromically shifted absorption and, in cases of fluorine substitution, ${ }^{19} \mathrm{~F}$-coupled electron-paramagnetic-resonance (EPR) spectra. By contrast, functional Tyr224-substituted variants generate radicals with unaltered light-absorption and EPR signatures as they produce verruculogen. The previously reported alternative product of the Tyr68Phe variant, which is also produced in competition with verruculogen by wild-type FtmOx1 in ${ }^{2} \mathrm{H}_{2} \mathrm{O}$ or with $2,3-\mathrm{F}_{2} \mathrm{Tyr}$, a poorer hydrogen donor than tyrosine, at position 68 , is identified as the 26 -hydroxy-21,27-endoperoxide product, likely formed via the C26-O-O adduct of the longer-lived $\mathrm{C} 26 \cdot$ with another $\mathrm{O}_{2}$ equivalent. The results highlight the considerable chemical challenges the enzyme must navigate in averting both oxygen rebound and a second $\mathrm{O}_{2}$ coupling to obtain verruculogen selectively over other possible products. 


\section{INTRODUCTION}

The human hormones prostaglandin $\mathrm{G}_{2}$ and $\mathrm{H}_{2}\left(\mathrm{PGG}_{2}\right.$ and $\left.\mathrm{PGH}_{2}\right)$, the plantderived malaria drug artemisinin, and several microbial natural products contain cyclic dialkylperoxide (endoperoxide) moieties that underpin their biological activities. ${ }^{1}$ Each case of enzymatic endoperoxide installation that has been studied to date involves capture of $\mathrm{O}_{2}$ between a carbon radical and nearby olefin of the substrate..$^{2-4}$ The radical forms by hydrogen atom $(\mathrm{H} \bullet)$ transfer $(\mathrm{HAT})$ from the substrate to an oxidized enzyme intermediate generated from or by an iron cofactor. The longest-known and most extensively studied endoperoxide-installing enzymes are the cyclooxygenases (COX), which convert arachidonic acid to $\mathrm{PGG}_{2}$ and $\mathrm{PGH}_{2}{ }^{2}$ and are targets of nonsteroidal anti-inflammatory drugs. ${ }^{5}$ Two more recently identified endoperoxide synthases belong to the iron(II)- and 2-oxoglutarate-dependent (Fe/2OG) enzyme family. 3,4,6,7 Verruculogen synthase [also known as fumitremorgin B (ftnB) endoperoxidase or FtmOx1; EC 1.14.11.38] from Aspergillus fumigatus installs an endoperoxide between carbons 21 and 27 of $f \operatorname{tn} B$ ( 1 in Figure $1 A$ ), producing verruculogen (2 in Figure $1 \mathrm{~A}$ ) on the pathway to the tremor-inducing mycotoxin, fumitremorgin A. ${ }^{3} \mathrm{Nvfl}$ from Aspergillus novofumigatus IBT 16806 converts its substrate asnovolin $A$ to fumigatonoid $A$ by installing both (i) an endoperoxide between methyl (C13) and olefinic (C2') carbons and (ii) a hydroxyl group on C3' - reportedly in a single step. $^{8}$

In the COX reaction, two HAT steps, from $\mathrm{C} 13$ and to a peroxyl radical on C15, initiate and terminate turnover. ${ }^{2}$ These steps bracket three $\mathrm{C}-\mathrm{O}$-bond-forming radical couplings that capture two $\mathrm{O}_{2}$ molecules to install the endo- and hydro-peroxides of the 
PGG2 product. A single tyrosine (Tyr; three-letter abbreviations are used for clarity throughout the main text, whereas one-letter abbreviations, $Y$ and F, are used for space economy in some figures and in naming variants with non-canonical analogs) mediates both HATs. This Tyr residue is oxidized to a quasi-stable radical (Tyr•) in a peroxidasetype reaction between the enzyme's heme cofactor and the hydroperoxide of the initial $\mathrm{PGG}_{2}$ product, which is concomitantly reduced to the hydroxyl-bearing $\mathrm{PGH}_{2}$. The resulting Tyr accepts $\mathrm{H} \bullet$ from $\mathrm{C} 13$ and is regenerated by donating $\mathrm{H} \bullet$ to the $\mathrm{C} 15-\mathrm{O}-\mathrm{O} \bullet$ intermediate. The reaction thus proceeds catalytically until and unless the Tyr is adventitiously reduced, in which case it must be regenerated by another reaction of the heme with $\mathrm{PGG}_{2}$.

The first biophysical analysis of FtmOx1 proposed a similar catalytic role for a Tyr - - in this case generated from Tyr224 (Figure 1B) by the oxoiron(IV) (ferryl) intermediate characteristic of the $\mathrm{Fe} / 2 \mathrm{OG}$ enzyme class (Figure 1C, pink arrows). ${ }^{3}$ Evidence included an x-ray crystal structure of the enzyme in complex with 20G, but lacking the prime substrate, 1 (Figure 1B), and transient ultraviolet-visible (UV-vis) absorption and electron paramagnetic resonance (EPR) spectra from the reaction of the complete $\mathrm{FtmOx} 1 \cdot \mathrm{Fe}(\mathrm{II}) \cdot 2 \mathrm{OG} \cdot 1$ complex with $\mathrm{O}_{2}$. To the best of our knowledge, the proposed COX-like catalytic turnover after a single ferryl-formation event has not been demonstrated in an Fe/2OG enzyme. ${ }^{9}$ Both the above study and subsequent work by our group ${ }^{10}$ provided evidence that Tyr224 is dispensable for production of 2, which would be unexpected for a residue mediating the crucial initiating HAT step. By contrast, our study posited HAT directly from C21 to the canonical ferryl complex - generated stoichiometrically in each turnover - and participation of a different tyrosine (Tyr68, 
Figure $1 \mathrm{~B}$ ) only as $\mathrm{H} \cdot$ donor to complete production of 2 (Figure $1 \mathrm{C}$, orange arrows). ${ }^{10}$ The obvious analogy of the initiating and terminating HAT steps to those previously demonstated in the stereoinversion reaction of carbapenem synthase $(\mathrm{CarC})^{11}$ led us to characterize this mechanism as a "CarC-like" pathway. Incorporation of ${ }^{18} \mathrm{O}$ from ${ }^{18} \mathrm{O}_{2}$ into the prenal co-product from the conversion of 1 to 4 (Figure 1C, cyan arrow), which occurs in an $\left[\mathrm{O}_{2}\right]$-dependent partition with the primary $\mathbf{1} \rightarrow \mathbf{2}$ reaction, implied competitive "oxygen rebound"12 and therefore proximity of C21 to the iron cofactor (Figure 1A). The Tyr224Phe variant exhibited reaction kinetics and verruculogen yields similar to those of wild-type FtmOx1, whereas the Tyr68Phe variant failed to accumulate the initial Tyr and generated an (unidentified) altered product along with very little verruculogen. Although the first study was subsequently editorially retracted, ${ }^{13}$ the statement of retraction noted only that the original conclusions were now judged to be insufficiently well-supported by the data; it did not cite the second study ${ }^{10}$ nor discuss its evidence for the conflicting interpretation. Moreover, the retraction was endorsed by only a single author of this first study, whereas the other authors explicitly stood by its conclusions, ${ }^{13,14}$ creating an imperative to seek more direct evidence for the roles of specific Tyr residues and the mechanisms of the required HAT steps. Very recently, while the study presented here was in preparation, an x-ray crystal structure of the FtmOx1•Fe(II)•2OG・1 complex was finally reported. ${ }^{4}$ Although its findings - including (1) proximity of $\mathrm{C} 21$ to the cofactor $(4.6 \AA)$, (2) proximity of the Tyr68 phenolic oxygen $\left(\mathrm{O}^{7}\right)$ to C26 (4.0 $\AA$ ), and (3) greater separation of Tyr224 $O^{7}$ from the cofactor $(8.5 \AA)$ than in the prior structures of the $\mathrm{FtmOx} 1 \cdot \mathrm{Fe}(\mathrm{II}) \cdot 2 \mathrm{OG}$ ternary complex $(4.2 \AA)^{3,4,10}$ - are obviously more consistent with the CarC-like mechanism of the second study than with 
the COX-like mechanism of the first, the structural work provided no direct evidence for the sites of the pair of Tyr radicals observed in the transient-state kinetics experiments.

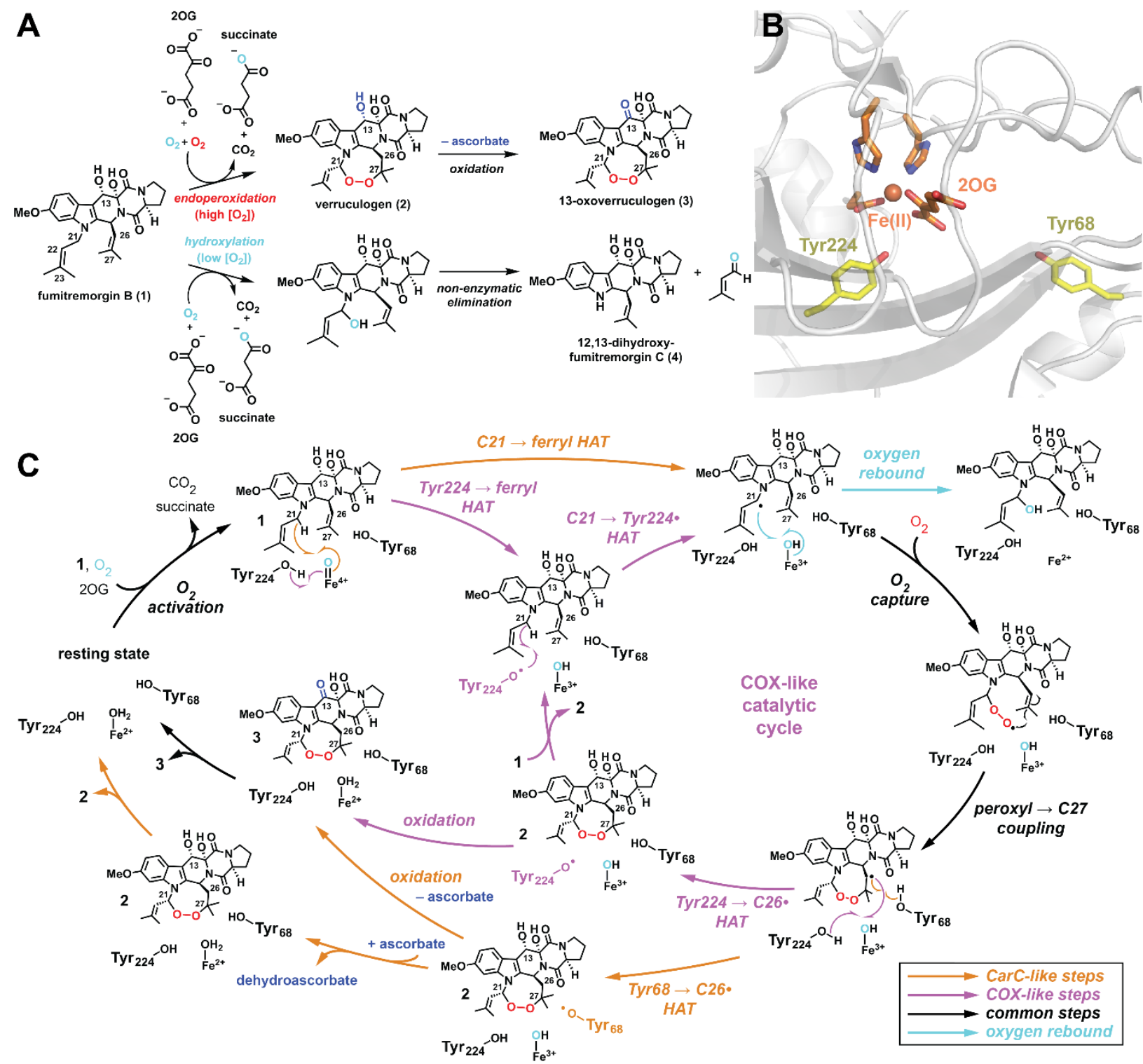

Figure 1. Structure and function of verruculogen synthase (Ftm0x1). (A) Two distinct reaction(s) catalyzed by FtmOx1 in an $\left[\mathrm{O}_{2}\right]$-dependent partition. (B) Active site of the FtmOx1 ternary complex (PDB entry: 4Y5S). ${ }^{3}$ The facial triad, iron(II), and 2OG are shown in orange, and the two contentious Tyr residues that were proposed to be critical for catalysis are highlighted in yellow. ${ }^{3,10}$ The corresponding ftnB-bound quaternary complex structure ${ }^{4}$ is shown in Figure $\mathbf{S 1}$. (C) The proposed mechanisms for substrate endoperoxidation and hydroxylation by FtmOx1. The COX-like mechanism ${ }^{2,3}$ follows the pink and black arrows, whereas the CarC-like mechanism, ${ }^{10,11}$ involving direct $\mathrm{C} 21 \rightarrow$ ferryl and Tyr68 $\rightarrow$ C26. HAT steps, follows the orange and black arrows. The two 
mechanisms differ by the identities of the $\mathrm{H} \cdot$ abstractor and donor that generates and quenches the substrate radical, respectively, to complete endoperoxidation. The steps after oxygen rebound (cyan arrow) leading to deprenylation are omitted for clarity. Because there are several possible points at which the co-product, succinate, could dissociate, it is depicted for the sake of simplicity as departing in the $\mathrm{O}_{2}$-activation step, even though it likely remains bound at least until after the ferryl complex abstracts $\mathrm{H} \bullet$.

Here, we have used the methodology pioneered by Schultz and coworkers ${ }^{15}$ to replace either of the two tyrosines (Tyr68 and Tyr224, Figure 1B) with a non-canonical Tyr analog: 3-fluorotyrosine (3-FY), 2,3-difluorotyrosine (2,3- $\left.\mathrm{F}_{2} \mathrm{Y}\right)$, 3,5-difluorotyrosine (3,5- $\left.\mathrm{F}_{2} \mathrm{Y}\right)$, 3-chlorotyrosine (3-CIY), or 4-aminophenylalanine (4- $\left.\mathrm{NH}_{2} \mathrm{~F}\right)$ (Figure S2). UVvis absorption and EPR spectra from the reactions of the variant proteins and analysis of the resultant organic products provide direct physical evidence that the Tyr that forms concomitantly with 2 resides on Tyr68 rather than Tyr224. The product analysis further implies that delay of the C26•-quenching HAT step by the presence of either a HAT incompetent or less facilely $\mathrm{H} \cdot$-donating Tyr analog (Figure S2) at position 68 or deuterium (from solvent) on the Tyr68 phenol enables a COX-like radical capture of a second $\mathrm{O}_{2}$ molecule, ultimately resulting in formation of a C26-hydroxylated endoperoxide-bearing product analogous to $\mathrm{PGH}_{2}{ }^{2}$ and fumigatonoid $\mathrm{A} .^{8}$

\section{MATERIALS and METHODS}

Construction of FtmOx1 expression vectors and aminoacyl-tRNA synthase plasmids. The E. coli codon-optimized wild-type gene sequence of Aspergillus fumigatus FtmOx1 that ends with an amber stop codon (TAG) was inserted into pET$28 \mathrm{a}$ between the $\mathrm{Ndel}$ and $\mathrm{BamHl}$ retriction sites, as previously described. ${ }^{10} \mathrm{An} \mathrm{N}$ terminal hexahistidine tag for nickel binding followed by a linker sequence was included to facilitate protein purification. The wild-type DNA sequence is as follows: 
TCTGCTGGAGGAAGATGGTGCGTTCATCCTGAAGGGCCTGCTGCCGTTCGACGTGGTTGAGAGC TTTAACCGTGAACTGGATGTGCAGATGGCGATCCCGCCGCCGAAAGGCGAGCGTCTGCTGGCGG ACAAGTACCCGCCGCACTTCAAATATGTGCCGAACGTTGCGACCACCTGCCCGACCTTTCGTAA CACCGTGCTGATCAACCCGGTTATCCACGCGATTTGCGAAGCGTACTTCCAACGTACCGGCGAT TATTGGCTGAGCGCGGCGTTTCTGCGTGAGATTGAAAGCGGTATGCCGGCGCAGCCGTTTCACC GTGACGATGCGACCCACCCGCTGATGCACTATCAGCCGCTGGAGGCTCCGCCGGTTAGCCTGAG CGTTATCTTCCCGCTGACCGAGTTTACCGAGGAAAACGGCGCGACCGAAGTTATTCTGGGTAGC CATCGTTGGACCGAGGTGGGTACCCCGGAACGTGATCAAGCGGTTCTGGCGACCATGGACCCGG GTGATGTGCTGATCGTTCGTCAACGTGTGGTTCATGCGGGTGGCGGTAACCGTACCACCGCGGG CAAGCCGCGTCGTGTGGTTCTGGCGTACTTCAACAGCGTGCAGCTGACCCCGTTTGAAACCTAT CGTACCATGCCGCGTGAGATGGTGGAAAGCATGACCGTTCTGGGCCAACGTATGCTGGGTTGGC GTACCATGAAACCGAGCGATCCGAACATCGTTGGTATTAACCTGATTGATGACAAGCGTCTGGA AAATGTTCTGCAACTGAAAGCGGCGGACAGCCCGGCGTAG

where the codons for Tyr68 and Tyr224 are shown in bold. The corresponding protein sequence is:

MGSSHHHHHHSSGLVPRGSHMTVDSKPQLQRLAADADVDRMCRLLEEDGAFILKGLLPFDVVES FNRELDVQMA I PP PKGERLLADKYPPHFKYVPNVATTCPTFRNTVLINPVIHAICEAYFQRTGD YWLSAAFLREIESGMPAQPFHRDDATHPLMHYQPLEAPPVSLSVIFPLTEFTEENGATEVILGS HRWTEVGTPERDQAVLATMDPGDVLIVRQRVVHAGGGNRTTAGKPRRVVLAYFNSVQLTPFETY RTMPREMVESMTVLGQRMLGWRTMKPSDPNIVGINLIDDKRLENVLQLKAADSPA

with Tyr68 and Tyr224 shown in bold. The N-terminal methionine is excised upon expression in E. coli (Table S3). ${ }^{16}$

To perform site-specific non-canonical amino acid (ncAA) incorporation, the codons corresponding to Tyr68 and Tyr224 were individually changed to amber stop codons. The original terminating stop codon of the wild-type sequence, an amber stop codon, was also converted to an ochre stop codon (TAA). Both replacements were achieved by the polymerase chain reaction (PCR) with primer pairs including Bsal recognition sites (underlined):

Y68TAG forward: 5' -GGAGGTCTCACAAGTAGCCGCCGCACTTCAAATATG-3' 
Y68TAG reverse: 5' -GGAGGTCTCACTTGTCCGCCAGCAGAC-3'

Y224TAG forward: 5'-GGAGGTCTCACTGGCGTAGTTCAACAGCGTGCA-3'

Y224TAG reverse: 5' -GGAGGTCTCACGCCAGAACCACACGAC-3'

Stop codon forward: 5' -GGAGGTCTCACCGGCGTAAGGATCCG-3'

Stop codon reverse: 5' -GGAGGTCTCACCGGGCTGTCC-3'

Digestion of the PCR products with Bsal and ligation with T4 ligase afforded the correct plasmids, which were verified by sequencing at the Penn State University Genomics Core Facility (University Park, PA). The 5 ncAAs were incorporated using three different aminoacyl-tRNA synthases (aaRSs) and their corresponding tRNACUA. Specifically, fluorotyrosines (3-FY, 3,5- $\mathrm{F}_{2} \mathrm{Y}$, and $2,3-\mathrm{F}_{2} \mathrm{Y}$ ) were incorporated using $\mathrm{F}_{\mathrm{n}} \mathrm{Y}-\mathrm{RS}$ E3 encoded within the pEVOL plasmid, kindly provided by Professor JoAnne Stubbe at MIT. ${ }^{17}$ 3-CIY was incorporated using CIY-RS expressed from the pEVOL plasmid developed by Professor Jiangyun Wang at the Chinese Academy of Sciences. ${ }^{18} 4-\mathrm{NH}_{2} \mathrm{~F}$ incorporation was originally attempted via pDule-para-aminoPhe from Professor Ryan Mehl at Oregon State University ${ }^{19}$, but this route yielded only Tyr $\rightarrow$ Phe variants in poor yield as a result of background contamination (Table S3). It was instead accomplished by exploiting the polyspecificity of the aaRS in pUltra-CNF from Professor Peter Schultz at The Scripps Research Institute ${ }^{20}$ to incorporate 4-azidophenylalanine (4-AzF) and subsequently reductively cleaving the azido moiety with tris(2-carboxyethyl)phosphine (TCEP) during purification (vide infra). ${ }^{21}$

Synthesis of fluorinated tyrosine analogs. The enzymatic synthesis has been reviewed elsewhere. ${ }^{22}$ All chemicals were purchased from Sigma-Aldrich, unless otherwise stated. The substituted phenol (10 mmol of either 2-fluorophenol, 2,6difluorophenol, or 2,3-difluorophenol, yielding 3-FY, 3,5- $\mathrm{F}_{2} \mathrm{Y}$, or $2,3-\mathrm{F}_{2} \mathrm{Y}$, respectively) was incubated at $\mathrm{pH} 8.25$ for 2-3 weeks at ambient temperature in the dark in a one-liter reaction containing tyrosine phenol lyase (TPL) at $A_{280}=0.65,16.6 \mathrm{~g}$ ammonium acetate, $7 \mathrm{~mL}$ pyruvic acid, $10 \mathrm{~mL}$ 25-30\% ammonium hydroxide (VWR), $27.4 \mathrm{mg}$ pyridoxal 5'-phosphate hydrate, and $0.35 \mathrm{~mL}$ 2-mercaptoethanol. For the 3-FY-yielding 
reaction, $0.45 \mathrm{~mL}$ of the monofluorophenol was added daily, while for the $3,5-\mathrm{F}_{2} \mathrm{Y}$ and 2,3- $\mathrm{F}_{2} \mathrm{Y}$ reactions, $0.65 \mathrm{~g}$ of the appropriate difluorophenol was added every other day until a total of $30 \mathrm{mmol}$ phenol had been added. The reaction mixture was applied to a column with $~ 300 \mathrm{~mL}$ Dowex 50WX8 (hydrogen form) 50-100 mesh cation exchange resin in $3 \mathrm{M}$ hydrochloric acid (Fisher). The column was washed with two volumes of deionized water and eluted with $\sim 500 \mathrm{~mL} 10 \%$ ammonium hydroxide solution. Ammonia and water were removed, and the volume was reduced to $30-50 \mathrm{~mL}$ under reduced pressure at $40{ }^{\circ} \mathrm{C}$. The resulting solution with precipitated tyrosine products was cooled at $4^{\circ} \mathrm{C}$ overnight and later centrifuged. The pellet was washed with cold deionized water to remove impurities and dried under vacuum. The identities of the tyrosine analogs were confirmed by ${ }^{1} \mathrm{H}$ - and ${ }^{19} \mathrm{~F}-\mathrm{NMR}$ spectra in $\mathrm{D}_{2} \mathrm{O}$ with a drop of trifluoroacetic acid (Fisher) as a ${ }^{19} \mathrm{~F}$ chemical shift reference $(-76.1 \mathrm{ppm})$ at room temperature. The NMR spectra (Figures S23-S25) are consistent with published spectra. ${ }^{23}$

3-FY: ${ }^{1} \mathrm{H}$ NMR $\left(400 \mathrm{MHz}, \mathrm{D}_{2} \mathrm{O}\right) \delta 6.9806\left(\mathrm{~d},{ }^{3} \mathrm{JHF}=11.9 \mathrm{~Hz}, 1 \mathrm{H}\right), 6.8820(\mathrm{~m}, 2 \mathrm{H}), 4.2098$ $\left(\mathrm{t},{ }^{3} \mathrm{JHH}=6.4 \mathrm{~Hz}, 1 \mathrm{H}\right), 3.1731\left(\mathrm{dd},{ }^{2} \mathrm{JHH}_{\mathrm{HH}}=14.7 \mathrm{~Hz},{ }^{3} \mathrm{JHH}_{\mathrm{HH}}=5.3 \mathrm{~Hz}, 1 \mathrm{H}\right), 3.0462\left(\mathrm{dd},{ }^{2} \mathrm{JHH}_{\mathrm{HH}}=\right.$ $\left.14.7 \mathrm{~Hz},{ }^{3} \mathrm{JHH}=7.7 \mathrm{~Hz}, 1 \mathrm{H}\right) ;{ }^{19} \mathrm{~F}$ NMR $\left(400 \mathrm{MHz}, \mathrm{D}_{2} \mathrm{O},{ }^{1} \mathrm{H}\right.$-decoupled) $\delta-136.9516$ (s).

\section{3,5- $\mathrm{F}_{2} \mathrm{Y}$ :}

${ }^{1} \mathrm{H}$ NMR $\left(400 \mathrm{MHz}, \mathrm{D}_{2} \mathrm{O}\right) \delta 6.9025\left(\mathrm{dd},{ }^{3} J_{\mathrm{HF}}=16.2 \mathrm{~Hz},{ }^{5} J_{\mathrm{HF}}=8.6 \mathrm{~Hz}, 2 \mathrm{H}\right), 4.2828(\mathrm{t}$, $\left.{ }^{3} J_{\mathrm{HH}}=6.4 \mathrm{~Hz}, 1 \mathrm{H}\right), 3.2407\left(\mathrm{dd},{ }^{2} J_{\mathrm{HH}}=14.8 \mathrm{~Hz},{ }^{3} J_{\mathrm{HH}}=5.4 \mathrm{~Hz}, 1 \mathrm{H}\right), 3.1151\left(\mathrm{dd},{ }^{2} J_{\mathrm{HH}}=\right.$ $\left.14.7 \mathrm{~Hz},{ }^{3} \mathrm{HH}=7.6 \mathrm{~Hz}, 1 \mathrm{H}\right) ;{ }^{19} \mathrm{~F}$ NMR $\left(400 \mathrm{MHz}, \mathrm{D}_{2} \mathrm{O},{ }^{1} \mathrm{H}\right.$-decoupled) $\delta-133.5832(\mathrm{~s})$.

\section{2,3- $\mathrm{F}_{2} \mathrm{Y}$ :}

${ }^{1} \mathrm{H} \mathrm{NMR}\left(400 \mathrm{MHz}, \mathrm{D}_{2} \mathrm{O}\right) \delta 6.8737\left(\mathrm{t},{ }^{3} \mathrm{HHH}_{\mathrm{HH}} \approx{ }^{5} \mathrm{JFF}_{\mathrm{HF}}=8.2 \mathrm{~Hz}, 1 \mathrm{H}\right), 6.7203\left(\mathrm{t},{ }^{3} \mathrm{JHH}_{\mathrm{HH}} \approx 5 \mathrm{JHF}_{\mathrm{HF}}=\right.$ $8.2 \mathrm{~Hz}, 1 \mathrm{H}), 4.2528\left(\mathrm{t},{ }^{3} \mathrm{JHH}_{\mathrm{HH}}=6.4 \mathrm{~Hz}, 1 \mathrm{H}\right), 3.2844\left(\mathrm{dd},{ }^{2} J_{\mathrm{HH}}=14.9 \mathrm{~Hz},{ }^{3} J_{\mathrm{HH}}=5.7 \mathrm{~Hz}\right.$, $1 \mathrm{H}), 3.1413\left(\mathrm{dd},{ }^{2} \mathrm{JHH}_{\mathrm{HH}} 14.9 \mathrm{~Hz},{ }^{3} \mathrm{JHH}=7.3 \mathrm{~Hz}, 1 \mathrm{H}\right) ;{ }^{19} \mathrm{~F} \mathrm{NMR}\left(400 \mathrm{MHz}, \mathrm{D}_{2} \mathrm{O},{ }^{1} \mathrm{H}-\right.$ decoupled) $\delta-141.5976\left(d,{ }^{3} J_{F F}=21.0 \mathrm{~Hz}\right),-161.5738\left(d,{ }^{3} J_{F F}=21.0 \mathrm{~Hz}\right)$.

Expression of FtmOx1 non-canonical variants. 3-Chloro-L-tyrosine (3-CIY), 4-aminoL-phenyalanine $\left(4-\mathrm{NH}_{2} \mathrm{~F}\right)$, 4-amino-L-phenyalanine hydrochloride $\left(4-\mathrm{NH}_{2} \mathrm{~F} \cdot \mathrm{HCl}\right)$, and 4azido-L-phenyalanine (4-AzF) were purchased from Chemlmpex International and used without further purification. The pET-28a plasmid carrying either the Y68TAG or 
Y224TAG substitution was used along with the appropriate aaRS plasmid to cotransform E. coli BL21(DE3) (New England BioLabs, catalogue number C2527H). The appropriate antibiotics were added into the culture medium at the subsequent growth and expression steps, as follows: kanamycin (50 mg/L, for pET-28a), chloramphenicol (35 mg/L, for $\mathrm{pEVOL}$, pre-dissolved in ethanol), tetracycline hydrochloride (10 mg/L, for pDule), and spectinomycin (50 mg/L, for pUltra). A seed culture was prepared by inoculating a single colony into $300 \mathrm{~mL}$ rich LB medium ( $35 \mathrm{~g} / \mathrm{L}$ tryptone, $20 \mathrm{~g} / \mathrm{L}$ yeast extract, and $5 \mathrm{~g} / \mathrm{L}$ sodium chloride (EMD)) in a 500-mL Erlenmeyer flask and was incubated overnight at $30^{\circ} \mathrm{C}$ with shaking at $180 \mathrm{rpm}$. The next day, a $20-\mathrm{mL}$ aliquot of the seed culture was transferred into each production culture, consisting of $1 \mathrm{~L}$ of rich LB medium in a $2800-\mathrm{mL}$ baffled flask. The production cultures were then incubated at $37^{\circ} \mathrm{C}$ with shaking at $200 \mathrm{rpm}$. When the $\mathrm{OD}_{600}$ reached $0.5-0.6,10 \mathrm{~mL}$ of $100 \mathrm{mM}$ ncAA stock, prepared by dissolving $1 \mathrm{mmol}$ of ncAA in $10 \mathrm{~mL}$ of deionized water supplemented with $160 \mu \mathrm{L} 8 \mathrm{M} \mathrm{NaOH}$ (Fisher), was added to each flask to give a final concentration of $1 \mathrm{mM}$. Induction of the amber suppression machinery was then initiated by addition of L-arabinose to a final concentration of $1 \mathrm{~g} / \mathrm{L}$ (Dot Scientific). For incorporation of $4-\mathrm{AzF}, 0.5 \mathrm{mmol}$ of the solid was instead directly added to each 1-L production culture to give a final concentration of $0.5 \mathrm{mM}$, and the induction step for pUltra was carried out later with isopropyl $\beta$-D-1-thiogalactopyranoside (IPTG; Gemini). The arabinose-induced cultures were then incubated at $30^{\circ} \mathrm{C}$ with the same shaking speed until they reached $\mathrm{OD}_{600}=0.8$, at which time IPTG was added to a final concentration of $0.25 \mathrm{~g} / \mathrm{L}$. The protein variants were subsequently expressed overnight (for $14-16$ hours) at $18^{\circ} \mathrm{C}$ at the same shaking speed (200 rpm).

Purification of FtmOx1 variants. The purification of FtmOx1 variants was carried out at $4{ }^{\circ} \mathrm{C}$ as described previously, ${ }^{10}$ with minor modifications noted below. The cells were collected at $6000 \mathrm{~g}$ for $12 \mathrm{~min}$, frozen in liquid nitrogen, and then weighed. Cell paste was resuspended in $3 \mathrm{~mL} / \mathrm{g}$ of lysis buffer containing $100 \mathrm{mM}$ Tris base (Dot Scientific) adjusted with $\mathrm{HCl}$ to $\mathrm{pH} 8.0$ (at $4{ }^{\circ} \mathrm{C}$ ). Lysozyme, deoxyribonuclease I, and phenylmethylsulfonyl fluoride (PMSF; Amresco) were added to final concentrations of 1 $\mathrm{mg} / \mathrm{mL}, 0.1 \mathrm{mg} / \mathrm{mL}$, and $1 \mathrm{mM}$, respectively. The cells were subsequently lysed by sonication (QSonica Q500; full power at $750 \mathrm{~W}$ with $20 \mathrm{kHz}$ frequency) on ice by a 28 - 
min cycle of $10 \mathrm{~s}$ on with $60 \%$ power and $30 \mathrm{~s}$ off. The resulting lysate was centrifuged at $22000 \mathrm{~g}$ for $30 \mathrm{~min}$, during which one fourth the lysate volume of nickel-nitrilotriacetate (Ni-NTA) agarose resin was equilibrated with 5 column volumes of lysis buffer. The supernatant was thoroughly mixed with the Ni-NTA resin and incubated for $\geq 30 \mathrm{~min}$. The resin was filtered and the liquid collected, followed by application of 10 column volumes of wash buffer [lysis buffer with $15 \mathrm{mM}$ imidazole (Beantown Chemical)] to the resin. The His-tagged protein was then eluted from the resin in 6 separate columnvolume fractions of elution buffer (lysis buffer with $250 \mathrm{mM}$ imidazole). Fractions were evaluated by SDS-PAGE (12\% polyacrylamide) stained with Coomassie Blue, and those fractions containing a significant quantity of the desired protein - typically the first three fractions - were pooled. The samples were concentrated with Amicon Ultra 10$\mathrm{kDa}$ MWCO centrifugal filters (Millipore) to $~ 20 \mathrm{~mL}$ and dialyzed overnight in 6-8 kDa MWCO (SpectraPor) tubing against $4 \mathrm{~L}$ of lysis buffer with $5 \mathrm{mM}$ EDTA (Dot Scientific) to remove metal ions. Two more rounds of dialysis were performed against $4 \mathrm{~L}$ lysis buffer. The purified protein was further concentrated to between 0.5 and $2 \mathrm{mM}$ with another set of centrifugal filters, and its concentration was determined by its absorbance at $280 \mathrm{~nm}$ using the molar absorption coefficient $\left(26,930 \mathrm{M}^{-1} \mathrm{~cm}^{-1}\right)$ estimated from the protein sequence ${ }^{24}$ assuming that the tyrosine analogs absorb as tyrosine. ${ }^{21}$ Incorporation of ncAAs was confirmed by SDS-PAGE analyses of eluted fractions compared with negative controls expressed in media without ncAAs (Figures S26-S29). The final sample was exchanged into deionized water and analyzed with electrospray ionization mass spectrometry (ESI-MS; ThermoFisher Q Exactive Hybrid QuadrupoleOrbitrap) following liquid chromatography (LC; ThermoFisher Vanquish Flex UHPLC) at the Penn State University Proteomics and Mass Spectrometry Core Facility (University Park, PA) (Figures S30-S34). The $\mathrm{m} / \mathrm{z}$ values of ions with greatest abundance from the resulting spectra were input into ESIprot ${ }^{25}$ to determine the average molecular mass (Table S3). The concentrated protein samples were stored at $4{ }^{\circ} \mathrm{C}$ and used for experiments within two weeks of purification. The amber suppression efficiency ranged from 5\% [for $\mathrm{Y} 224\left(2,3-\mathrm{F}_{2} \mathrm{Y}\right)$ ] to $27 \%$ [for $\mathrm{Y} 68(3-\mathrm{ClY})$ ] in successful attempts. Y224 noncanonical variants invariably showed poorer yields than the $Y 68$ counterparts, with the worst cases being the variants with fluorinated tyrosines (Figures S26-S29). 
Incorporated 4-AzF was reduced to $4-\mathrm{NH}_{2} \mathrm{~F}$ after elution, during concentration, and prior to overnight dialysis. Specifically, a $0.5 \mathrm{M}$ stock of TCEP was prepared by dissolving $1.43 \mathrm{~g}$ of TCEP in $10 \mathrm{~mL}$ cold deionized water and adjusting to $\mathrm{pH} 7.0$ with aqueous $\mathrm{NaOH}$. The pooled fractions from the $\mathrm{Ni}(\mathrm{II})-\mathrm{NTA}$-agarose affinity column were brought to $10 \mathrm{mM}$ TCEP and incubated at $4{ }^{\circ} \mathrm{C}$ for $\geq 3 \mathrm{~h}$. Given the protein's ability to bind its rather large substrate (ftnB, 1), it was anticipated (correctly) that TCEP would be able to access positions 68 (on a peripheral loop) and 224 (near the iron site). Excess TCEP was subsequently removed by multiple rounds of dialysis. Because $4-\mathrm{NH}_{2} \mathrm{~F}$ has a mass nearly identical to that of tyrosine, protein samples were subjected to mass spectrometric analysis both prior and subsequent to the TCEP treatment to verify complete reduction of the encoded $4-\mathrm{AzF}$ to the desired $4-\mathrm{NH}_{2} \mathrm{~F}$ (Table S3 and Figure S34).

Preparation of buffers for protein characterization. Unless stated otherwise, the following experiments were performed in the lysis buffer (100 mM Tris, $\mathrm{pH} 8.0$ at $4{ }^{\circ} \mathrm{C}$ ). For the pD 8.0 buffer, 100 mM HEPES in $\mathrm{D}_{2} \mathrm{O}(99.9$ atom \% D) was titrated with $\mathrm{NaOH}$ in $\mathrm{D}_{2} \mathrm{O}$ to achieve an apparent $\mathrm{pH}$ of 7.84 measured by a glass electrode, based on the conversion formula $\mathrm{pH}_{\mathrm{app}}=0.929 \mathrm{pD}+0.41 .{ }^{26}$ The final atom percentage of deuterium in the $\mathrm{D}_{2} \mathrm{O}$ buffer was estimated to be $>96 \%$. It was verified for wild-type FtmOx1 that reactions carried out in Tris and HEPES buffers at $\mathrm{pH} 8.0$ gave mutually consistent kinetic results.

Deoxygenation and oxygenation of proteins and buffers. Proteins were exchanged into the $100 \mathrm{mM}$ Tris, $\mathrm{pH} 8.0$ buffer with centrifugal filters. The proteins were then placed in flasks and deoxygenated on a Schlenk line through three rounds of ten cycles of gentle evacuation (to $\sim 50 \mathrm{mmHg}$ for 3 seconds) and refilling with oxygen-free argon gas, with each round of gas exchange followed by at least 30 min incubation on ice under $800-820 \mathrm{mmHg}$ of argon with slow stirring. The $100 \mathrm{mM}$ Tris, pH 8.0 buffer ( $40 \mathrm{~mL}$ ) was also deoxygenated and oxygenated by similar evacuation-refilling cycles under argon and oxygen, respectively, except that each evacuation period was carried out for 20 seconds at $5-12 \mathrm{mmHg}$ with more vigorous stirring. The fully oxygenated $\mathrm{pD}$ 8.0 buffer was also prepared by this procedure. The ${ }^{18} \mathrm{O}_{2}$-saturated buffer was prepared 
by five 10-min evacuation-refilling (with argon) cycles with $2 \mathrm{~mL}$ of the desired buffer in a Schlenk flask on ice, followed by a final evacuation period for another $5 \mathrm{~min}$. The flask was then charged to 820 Torr with ${ }^{18} \mathrm{O}_{2}(g)$ (99 atom \% ${ }^{18} \mathrm{O}$; Sigma-Aldrich). The buffer inside the flask was allowed to reach equilibrium with the ${ }^{18} \mathrm{O}_{2}(\mathrm{~g})$ for $30 \mathrm{~min}$ prior to being used. Processed buffers and protein samples were subsequently brought into an MBraun (Peabody, MA) LabMaster 100 anoxic glovebox and placed on a cold block maintained at $5{ }^{\circ} \mathrm{C}$, ready for the experiments.

UV-vis absorption spectroscopy to monitor 20G binding. Aliquots of $0.25 \mathrm{~mL}$ containing $0.70 \mathrm{mM}$ wild-type FtmOx1 or a variant and $0.56 \mathrm{mM} \mathrm{Fe}\left(\mathrm{NH}_{4}\right)_{2}\left(\mathrm{SO}_{4}\right)_{2}$ in 100 $\mathrm{mM}$ Tris, $\mathrm{pH} 8.0$ were prepared in a $1.7-\mathrm{mL}$ microcentrifuge tube at room temperature. The mixtures were then transferred to a $160-\mu \mathrm{L}$ quartz cuvette with a $1-\mathrm{cm}$ path length, placed in an Agilent 8453 UV-visible spectrophotometer, and used as blanks before $2 \mathrm{OG}$ addition. A stock solution of $50 \mathrm{mM} 2 \mathrm{OG}$ was freshly made by dissolving 2oxoglutaric acid disodium salt dihydrate solid in the $100 \mathrm{mM}$ Tris, $\mathrm{pH} 8.0$ buffer. To reach a final concentration of $1.2 \mathrm{mM} 2 \mathrm{OG}$ ( 2.1 equivalents relative to iron cofactor), which should result in nearly saturated 2OG binding behavior for wild-type FtmOx1 according to Yan et al., ${ }^{3} 6 \mu \mathrm{L}$ of the $20 \mathrm{G}$ stock solution was added to the protein samples in the cuvette, and the corresponding UV-visible absorption spectra were recorded.

LC-MS analysis of Ftm Ox1 reaction products. $1 \mu \mathrm{L}$ aliquots of $1 \mathrm{mM}$ wild-type FtmOx1 or a variant (adjusted accordingly for variants with poorer solubility), $1 \mathrm{mM}$ $\mathrm{Fe}\left(\mathrm{NH}_{4}\right)_{2}\left(\mathrm{SO}_{4}\right)_{2}$ (EM Science), $100 \mathrm{mM} 2 \mathrm{OG}$, and $100 \mathrm{mM}$ sodium ascorbate (Spectrum Chemical) were mixed (in the specific order given) at the bottom of $1.7-\mathrm{mL}$ microcentrifuge tubes. A 2- $\mu \mathrm{L}$ aliquot of $25 \mathrm{mM} 1$ [Quality Phytochemicals; dissolved in dimethyl sulfoxide (DMSO; EM Science)] was pipetted to the side of the tubes to avoid undesired precipitation of proteins and co-substrates caused by DMSO. The drop of 1 was then flushed to the bottom using a syringe loaded with enough $\mathrm{O}_{2}$-saturated 100 mM Tris, pH 8.0 buffer (100 mM HEPES, pD 8.0 instead in the solvent deuteration experiment) to yield a final volume of $100 \mu \mathrm{L}$. The final concentrations were $10 \mu \mathrm{M}$ enzyme, $10 \mu \mathrm{M} \mathrm{Fe}(\mathrm{II}), 1 \mathrm{mM} 2 \mathrm{OG}, 1 \mathrm{mM}$ ascorbate, $0.50 \mathrm{mM} \mathrm{1}$, and $\sim 1.8 \mathrm{mM} \mathrm{O}_{2}$. 
Proton-deuteron exchange should be much faster $\left(>100 \mathrm{~s}^{-1}\right)^{27}$ for relatively solventexposed tyrosines (including Tyr68 and Tyr224) than Tyr• formation in the reaction ( $\left.10 \mathrm{~s}^{-1}\right)$. The tubes were immediately capped, removed from the anoxic chamber, and opened to the air for $\sim 1 \mathrm{~h}$ at ambient temperature, with gentle stirring to replenish the consumed $\mathrm{O}_{2}$. The reaction mixture was quenched by extracting twice with an equal volume $(100 \mu \mathrm{L})$ of HPLC-grade 1:1 (v/v) $n$-hexane:ethyl acetate (both from Alfa Aesar) solution. The combined organic layer was dried under $\mathrm{N}_{2}$ overnight and redissolved in $50 \mu \mathrm{L} \mathrm{HPLC}$-grade acetonitrile (EMD). The solution was then centrifuged at $14,000 \mathrm{~g}$ for 10 minutes to remove protein prior to sample injection on an Agilent 6410 Triple Quadrupole LC-MS instrument.

Assay samples $(2 \mu \mathrm{L})$ were injected onto an Agilent Zorbax Extend-C18 column $(4.6 \times 50 \mathrm{~mm}, 1.8 \mu \mathrm{m}$ particle-size $)$ in $70 \%$ solvent $A(0.1 \%$ formic acid in water) and $30 \%$ solvent $B$ (acetonitrile). A flow rate of $0.3 \mathrm{~mL} / \mathrm{min}$ was used throughout. The elution method started with a linear gradient from $70 \%$ to $0 \%$ solvent $A$ between 0 and 32 min following sample injection. The column was returned to $70 \%$ solvent $A$ through another linear gradient from 32 to $35 \mathrm{~min}$ and finally washed with $70 \%$ solvent $A$ from $35 \mathrm{~min}$ to $43 \mathrm{~min}$ before the next injection. Note that this method is identical to the one previously described, ${ }^{10}$ but with a diminished flow rate (instead of $0.8 \mathrm{~mL} / \mathrm{min}$ ). 1 and 1-derived products were detected at the $\mathrm{m} / \mathrm{z}$ values listed in Table $S 4$ by electrospray ionization in the positive-ion mode (ESI+) using the HPLC-MS instrument. Samples of 1 and 2 (Enzo Life Sciences; Farmingdale, NY) in acetonitrile served as external standards. The resulting total-ion and extracted-ion chromatograms (TIC and EIC) were analyzed and integrated using the Agilent MassHunter Quantitative Analysis Software. Certain precursor ions (those with $\mathrm{m} / \mathrm{z}$ values given in bold in Table S4) were selected for further product ion scans, in which the collision energy was set to $5 \mathrm{eV}$ and the scan range to $\mathrm{m} / \mathrm{z}=50$ to 526 .

For the ${ }^{18} \mathrm{O}_{2}$ incorporation experiments, the assay conditions and workup procedures were modified slightly. The reactions were carried out in duplicate using samples of $\mathbf{1}$ from two different commercial sources (Quality Phytochemicals or Cayman Chemical) under otherwise identical conditions. A $55-\mu \mathrm{L}$ solution containing 
$0.92 \mathrm{mM} \mathrm{FtmOx} 1$ (wild type or $\mathrm{Y} 68 \mathrm{~F}), 0.92 \mathrm{mM} \mathrm{Fe}\left(\mathrm{NH}_{4}\right)_{2}\left(\mathrm{SO}_{4}\right)_{2}$ (1 equivalent), $4.6 \mathrm{mM}$ $2 \mathrm{OG}$ (5 equivalents), and $0.46 \mathrm{mM}$ sodium ascorbate ( 0.5 equivalent) was placed in an $\mathrm{LC}$ autosampler glass vial. A $1 \mu \mathrm{L}$ aliquot of $25 \mathrm{mM} 1$ was added to the side of the vial. The vial was tightly sealed by an autosampler cap with a septum, and the drop of $\mathbf{1}$ was then flushed to the bottom with enough ${ }^{18} \mathrm{O}_{2}$-saturated $100 \mathrm{mM}$ Tris, $\mathrm{pH} 8.0$ buffer to give a final volume of $100 \mu \mathrm{L}$. The spatial segregation of the ternary complex from the substrate prior to ${ }^{18} \mathrm{O}_{2}$ addition also avoided unwanted reaction with the exclusively ${ }^{16} \mathrm{O}_{2}$ that might have been present from any residual atmospheric gases. The reactions were incubated for $3 \mathrm{~min}$ in the anoxic chamber, removed from the chamber, and either placed in boiling water for $3 \mathrm{~min}$ (for the analysis of 2OG and succinate) or quenched by two extractions with equal volumes $(100 \mu \mathrm{L})$ of HPLC-grade 1:1 (v/v) n-hexane:ethyl acetate solution (for the analysis of $\mathbf{1}$ and its derived products), with minimal exposure to the atmosphere. The samples for analysis of $\mathbf{1}$ and its reaction products were prepared as described above, except that only $25 \mu \mathrm{L}$ acetonitrile was used to dissolve the extracted material. The samples for succinate/2OG analysis were transferred to 1.7$\mathrm{mL}$ microcentrifuge tubes and centrifuged at $16,000 \mathrm{~g}$ for $10 \mathrm{~min}$ to remove the denatured protein. The supernatant was retained and subjected to filtration through a PALL 10K Omega centrifugal device with the same centrifuge settings, and the flowthrough was directly used for the LC-MS analysis.

For detecting $2 \mathrm{OG}$ and succinate, the assay samples $(4 \mu \mathrm{L})$ were injected onto an Agilent Zorbax Extend-C18 column in $95 \%$ solvent A ( $0.1 \%$ formic acid in water) and $5 \%$ solvent $B$ (acetonitrile). An isocratic elution spanning 5 minutes was then performed with a flow rate of $0.4 \mathrm{~mL} / \mathrm{min} .{ }^{28} 2 \mathrm{OG}$ and succinate were detected at the $\mathrm{m} / \mathrm{z}$ values listed in Table S4 by electrospray ionization in the negative-ion mode (ESI-) using the HPLC-MS instrument.

Stopped-flow absorption experiments. Stopped-flow absorption (SF-abs) experiments were performed on an Applied Photophysics (Surrey, UK) SX-19MV instrument housed in an MBraun LabMaster 100 anoxic chamber with a $1-\mathrm{cm}$ pathlength cell. The spectral data sets were acquired using a photodiode array (PDA) detector with a logarithmic time base, and the instrument was configured for a single 
equal-volume mix with a total drive volume of $\sim 120 \mu \mathrm{L}$. Deoxygenated $100 \mathrm{mM}$ Tris-Cl, $\mathrm{pH} 8.0$ buffer was used as the reference. Mixing of the syringe contents was pneumatically triggered by nitrogen gas at $125-130$ psi. One syringe was loaded with a solution of $0.40-0.56 \mathrm{mM}$ of wild-type FtmOx1 or a variant (with the exact concentrations noted in the legend to Figure S4), 0.8 equivalents $\mathrm{Fe}\left(\mathrm{NH}_{4}\right)_{2}\left(\mathrm{SO}_{4}\right)_{2}, 10$ equivalents 2OG, and 1.5 equivalents 1 in the $100 \mathrm{mM}$ Tris- $\mathrm{Cl}, \mathrm{pH} 8.0$ buffer; the solution was prepared by mixing its components in a $1.7-\mathrm{mL}$ microcentrifuge tube in the order noted and centrifuging at $14,000 \mathrm{~g}$ for $1 \mathrm{~min}$ to remove precipitate prior to loading. The other syringe was loaded with $\mathrm{O}_{2}$-saturated buffer. The syringe contents were allowed to reach thermal equilibrium with circulating water at $5{ }^{\circ} \mathrm{C}$ for at least 5 min prior to data acquisition. For experiments evaluating the accessibility/reactivity of tyrosyl radicals in FtmOx1 and its variants, $1 \mathrm{mM}$ sodium ascorbate was also included in the enzyme syringe.

Freeze-quench EPR experiments. Freeze-quench EPR (FQ-EPR) samples were prepared using an Update Instruments (Milwaukee, WI) quench-flow apparatus by mixing an equal volume of reactant solutions loaded in two 1-mL syringes and allowing the reaction to proceed for a desired time at $5{ }^{\circ} \mathrm{C}$ in the aging hose before quenching by injection into 2-methylbutane (isopentane) at $\sim-150{ }^{\circ} \mathrm{C}$. The frozen reaction solution was packed into an EPR tube to a desired height inside the cryo-solvent bath. Reaction times were chosen so that samples would be maximally enriched in the radical intermediates of interest. For continuous-wave (CW) X-band EPR measurements, the corresponding enzyme syringe for the $F Q$ apparatus was loaded from the material remaining in the stopped-flow enzyme syringe (with the exact concentrations noted in Figure S4 caption); the oxygen syringe was loaded with freshly prepared $\mathrm{O}_{2}$-saturated buffer. For pulsed Q-band experiments, higher radical concentrations were much preferred, and so the enzyme concentration was increased to $1.11 \mathrm{mM}$ in the enzyme syringe and the relative stoichiometries of the remaining components were maintained.

CW X-band $(\sim 9.4 \mathrm{GHz})$ EPR spectra at $30 \mathrm{~K}$ and $80 \mathrm{~K}$ were collected on a Magnettech MS5000X spectrometer equipped with an Oxford Instruments ESR900 continuous flow liquid He/liquid $\mathrm{N}_{2}$ cryostat and an Oxford Instruments ITC300 
temperature controller. Spectrometer conditions were set to $4 \mathrm{G}$ modulation field amplitude, $100 \mathrm{kHz}$ modulation frequency, and $31.6 \mu \mathrm{W}$ microwave power to avoid overmodulation and saturation. Pulse Q-band ( 34 GHz) echo-detected EPR measurements utilized a home-built intermediate-frequency extension $(+24.5 \mathrm{GHz})$ of a Bruker E580 X-band spectrometer equipped with an Oxford Instruments CF935P liquid He/liquid $\mathrm{N}_{2}$ cryostat, an Oxford Instruments ITC300 temperature controller, and a home-built Q-band resonator. ${ }^{29}$ The delay between the ( $\left.\pi / 2\right)$-pulse and $\pi$-pulse was set to $160 \mathrm{~ns}$. All pulse experiments were performed at $30 \mathrm{~K}$ using liquid $\mathrm{He}$ as cryogen to reach optimal relaxation rate for short shot-repetition times without significant loss of signal.

Chemical quench and product analysis. The chemical quench experiments were performed using the same quench-flow apparatus as for the FQ experiments, except that the aged reaction mixture $(\sim 50 \mu \mathrm{L})$ was instead quenched by injecting into a 1.7$\mathrm{mL}$ microcentrifuge tube containing $200 \mu \mathrm{L}$ GC-grade acetone (Burdick \& Jackson). The enzyme concentration was $0.56 \mathrm{mM}$ in the reactant syringe, as in the SF-abs experiments. The reaction was quenched at five reaction times: $10 \mathrm{~ms}, 50 \mathrm{~ms}, 350 \mathrm{~ms}$, $2 \mathrm{~s}$, and $10 \mathrm{~s}$, to follow the evolution of fumitremorgin B and fumitremorgin B-derived products along with the red-shifted tyrosyl radical at its half-maximum and maximum concentrations in addition to early and late time points. Duplicates were made for certain time points to ensure reproducibility. Precipitated protein was pelleted by centrifuging at $14,000 \mathrm{~g}$ for $10 \mathrm{~min}$, and the supernatant was removed, dried under $\mathrm{N}_{2}$ overnight, and redissolved in $50 \mu \mathrm{L}$ HPLC-grade acetonitrile. The procedure for the subsequent product analysis by LC-MS was identical to the procedure described above.

\section{RESULTS}

Tyr68 but not Tyr224 is the radical harboring site and the hydrogen donor. Incorporation of 3-fluorotyrosine (3-FY), 3,5-difluorotyrosine (3,5- $\left.\mathrm{F}_{2} \mathrm{Y}\right)$, , 2,3difluorotyrosine $\left(2,3-\mathrm{F}_{2} \mathrm{Y}\right)$, 3-chlorotyrosine (3-CIY), or 4-aminophenylalanine $\left(4-\mathrm{NH}_{2} \mathrm{~F}\right)$ at position 68 of FtmOx1 yielded variants with the ability to direct endoperoxide installation upon 1 to form 2 (Figure 2), by contrast to the previously noted ${ }^{10}$ redirection of the reaction to alternative product $\mathbf{5}$ by the canonical Y68F substitution (red trace in 
Figure 2A). This top-line observation confirmed our expectation that replacement of functional tyrosines with appropriate ncAAs would permit a finer dissection of the native reaction and roles of tyrosine residues therein.

For two of the Tyr224-substituted variants [Y224(3,5- $\left.\mathrm{F}_{2} \mathrm{Y}\right)$ and $\left.\mathrm{Y} 224\left(2,3-\mathrm{F}_{2} \mathrm{Y}\right)\right]$, however, we observed little to no product formation (green and purple traces in Figure $2 \mathrm{~B})$. On the basis of the inability of these variants to support development of either the 520-nm metal-to-ligand charge-transfer (MLCT) band characteristic of the FtmOx1•Fe(II)•2OG ternary complex (Figure S3C) or the appropriate transient absorption features (Figures S4F and S4L) upon subsequent rapid mixing of the presumptive quaternary complex with $\mathrm{O}_{2}$, we attribute this inactivity to a deficiency in proper 2OG binding. Indeed, structural modeling indicates that the appended fluorines could cause significant steric clashes in the tight 2OG-binding pocket (Figure S3B). We also noted that these Tyr224 $\rightarrow$ fluorotyrosine variants were more prone to precipitation upon concentration (Figure S3A), implying that the substitutions unexpectedly impair the enzyme's structural integrity. 


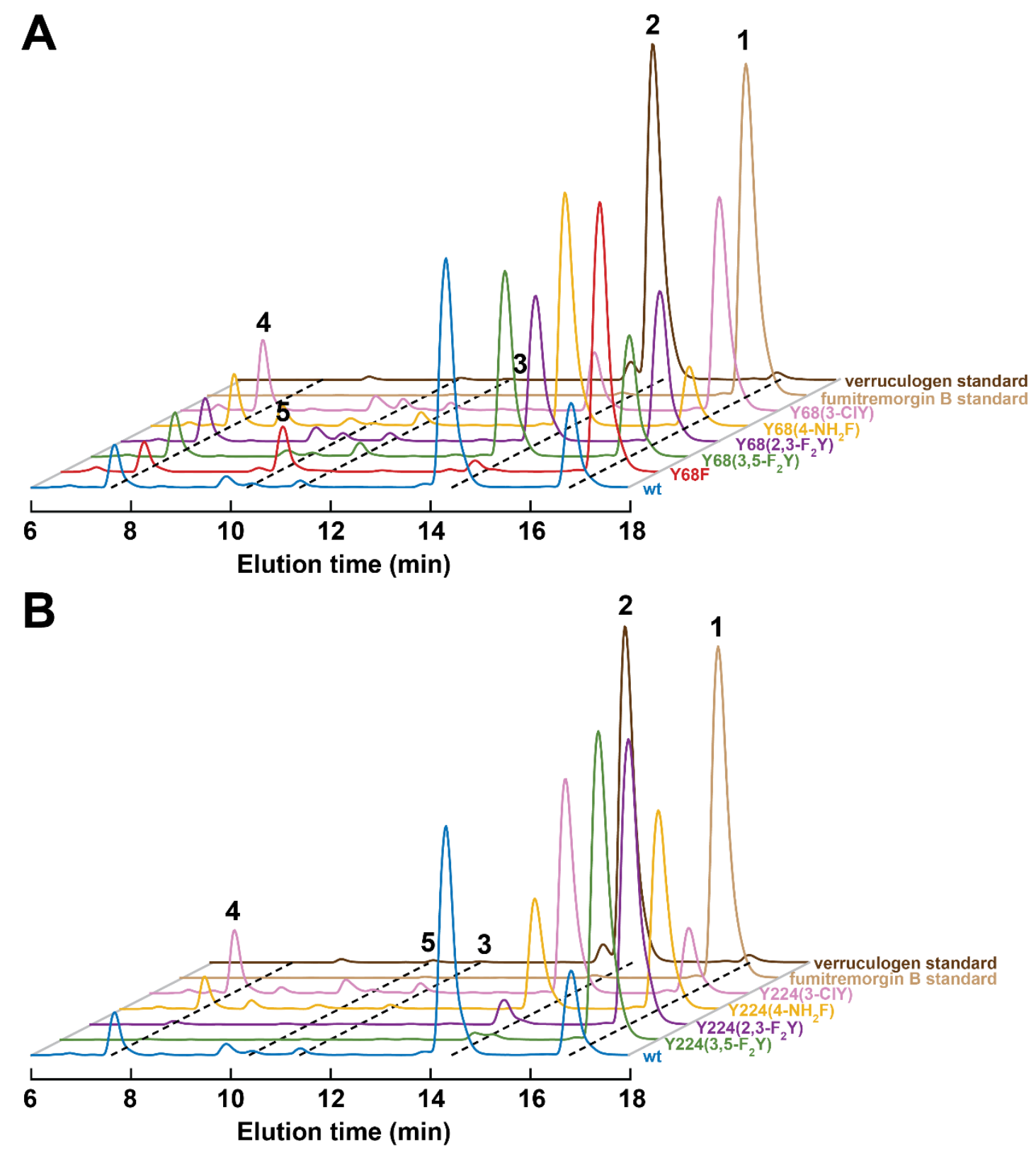

Figure 2. LC-MS chromatograms from the reactions of wild-type (wt) Ftm0x1 and its (A) Tyr68- and (B) Tyr224-substituted variants. The traces are color-coded as in Figure S2 according to the tyrosine analog at position 68 or 224. The reactions contained $10 \mu \mathrm{M}$ enzyme, $10 \mu \mathrm{M} \mathrm{Fe}(\mathrm{II}), 1 \mathrm{mM} 2 \mathrm{OG}, 1 \mathrm{mM}$ ascorbate, $0.50 \mathrm{mM}$ 1, and $1.8 \mathrm{mM} \mathrm{O}_{2}, \mathrm{pH} 8.0$ and were carried out at room temperature as described in Materials and Methods.

We next repeated aspects of the previously reported transient-state kinetic analysis of wild-type FtmOx1 to allow for the most direct comparison to the catalytic behavior of variants bearing a noncanonical Tyr analog. Mixing of the wild-type $\mathrm{FtmOx} 1 \cdot \mathrm{Fe}(\mathrm{II}) \cdot 2 \mathrm{OG} \cdot 1$ quaternary complex with an equal volume of $\mathrm{O}_{2}$-saturated buffer 
( $1.9 \mathrm{mM} \mathrm{O}_{2}$ ) resulted in development of the previously reported, ${ }^{3,10}$ broad, transient absorption band at $\sim 417 \mathrm{~nm}$ (Figures $3 \mathrm{~A}$ and S4A), which is significantly shifted from the usual $\sim 410 \mathrm{~nm}$ peak of tyrosyl radicals. This feature reached its maximum intensity at $0.35 \mathrm{~s}$ (Figure S5A). The $\mathrm{CW}$ X-band EPR spectrum at $30 \mathrm{~K}$ of a sample freezequenched at this reaction time exhibits the broad $g \approx 2$ signal seen in the earlier studies (Figure 3B). The fact that 2 was still readily detected (Figure 2) in reactions containing ascorbate, which prevents Tyr accumulation, presumably by rapidly reducing it (Figure S6), implies that the detected Tyr• is formed late in the reaction sequence and is not involved in the initiating HAT from C21 (orange arrows in Figure 1C). ${ }^{10}$ This conclusion is further corroborated by the results of chemical quench experiments (Figures S7 and S8), which showed that the Tyr develops as 2 forms; this temporal correspondence implies that the Tyr is generated by HAT to C26 to complete 2 formation. The chemical-quench results (Figures S7 and S8) also suggest that the previously reported subsequent dehydrogenation of $\mathbf{2}$ to $\mathbf{3}$ involves this Tyre, as the decay of the radical was seen to correlate temporally with formation of 3 (Figure 1A; Figure 1C, bottom left corner). This conclusion is validated by the observation that the yield of $\mathbf{3}$ was vastly diminished in the presence of ascorbate (Figures 2 and S6). ${ }^{10}$ This sequence of reactions - namely, hydrogen donation followed by desaturation - is analogous to the sequence of steps purportedly mediated by CarC. ${ }^{11}$

To identify the site of the Tyr•, we tested the variants bearing fluorinated tyrosines for the expected (1) hypsochromic shift in the sharp Tyr absorption feature and (2) a large hyperfine coupling $\left(A_{z} \approx 150 \mathrm{MHz}\right)$ from ${ }^{19} \mathrm{~F}$ ortho to the phenolic oxygen. ${ }^{17,30-34}$ We anticipated that, as has been seen in other enzymes, the minimally perturbed oxidation potentials of the 3-FY and 3,5- $\mathrm{F}_{2} \mathrm{Y}$ analogs ${ }^{33,35}$ (Figure S2) would make these fluorotyrosines, in particular, ideal probes. In SF-abs experiments on the $\mathrm{Y} 68(3-\mathrm{FY})$ and $\mathrm{Y} 68\left(3,5-\mathrm{F}_{2} \mathrm{Y}\right)$ variants, the characteristic feature of the Tyr developed with kinetics similar to those seen with the wild-type enzyme (Figures S4A-S4C and $\mathrm{S} 5 \mathrm{~A})$. Relative to the spectrum arising from the transient Tyr in the wild-type enzyme, which starts out somewhat bathochromically shifted $(\sim 417 \mathrm{~nm})$ from the band of most known protein-derived Tyr•s (408-412 nm), the spectra of the radical in the Y68(3-FY) and $Y 68\left(3,5-F_{2} Y\right)$ variants are indeed hypsochromically shifted to more usual maxima of 
411 (Figures $3 \mathrm{~A}$ and S4B) and $409 \mathrm{~nm}$ (Figures $3 \mathrm{~A}$ and S4C), respectively. The direction and magnitudes of these shifts from $417 \mathrm{~nm}$ are comparable to those seen in comparing the corresponding fluorinated tyrosyl radicals to unsubstituted Tyr ${ }^{\circ}$ in solution. ${ }^{33}$ The X-band EPR spectra at $30 \mathrm{~K}$ of samples freeze-quenched at a reaction time of $0.35 \mathrm{~s}$ exhibit prominent hyperfine couplings for the two fluorotyrosine-bearing variants that are not seen with wild-type FtmOx1 (Figures 3B and S9). The spectrum of the protein harboring the difluorinated analog is the broadest and exhibits couplings from two ${ }^{19} \mathrm{~F}$ nuclei, as expected (Figures $3 \mathrm{~B}$ and S9). These absorption and EPR spectra conclusively establish that the tyrosine (or fluorotyrosine) at position 68 donates hydrogen to form the previously detected accumulating radical.
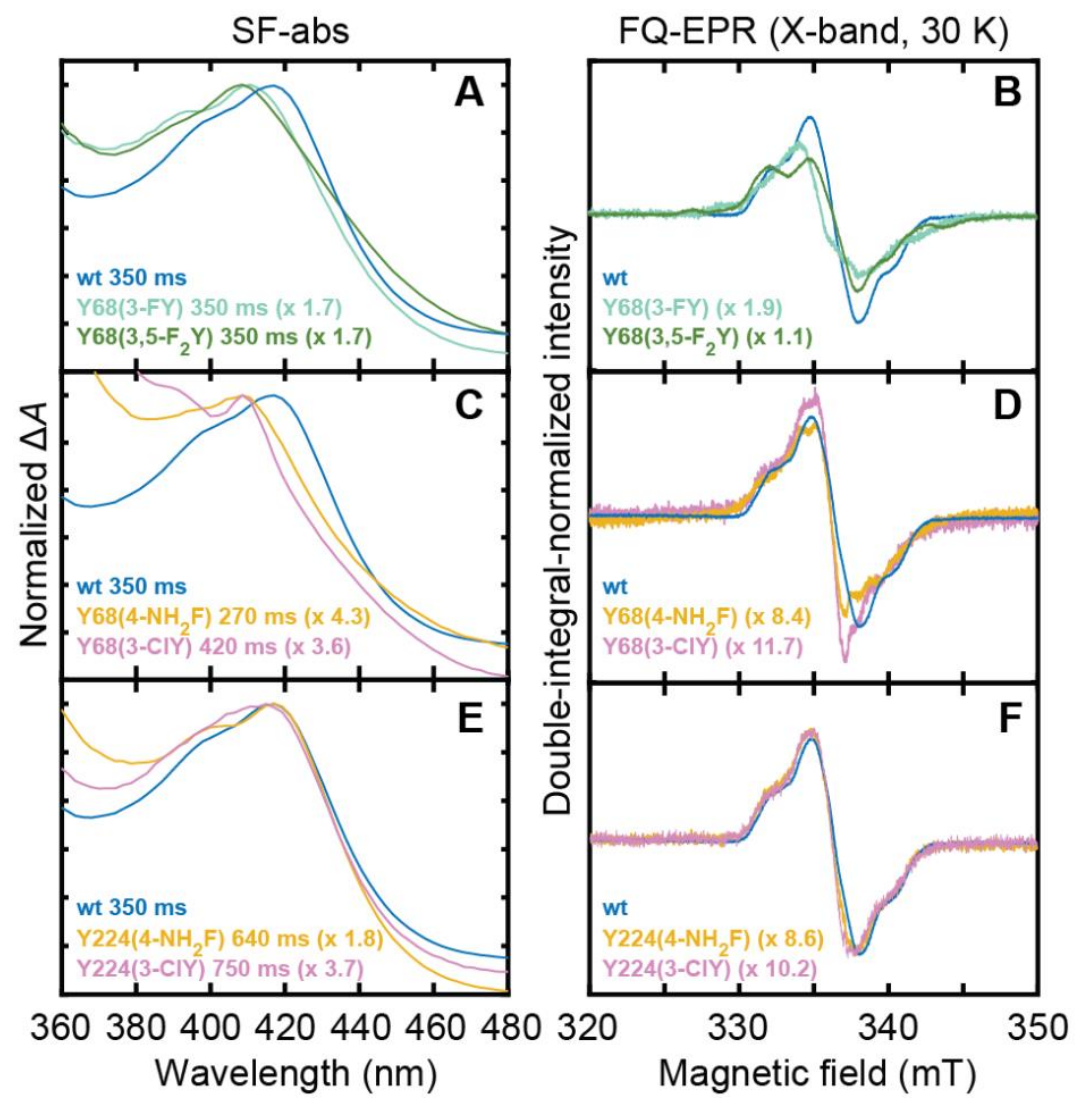

Figure 3. SF-abs (left) and X-band FQ-EPR spectra ( $30 \mathrm{~K}$, right) of the early radical intermediates in the reactions of wild-type (wt) FtmOx1 and variants that support their accumulation. $(\boldsymbol{A}, \boldsymbol{B})$ Spectra from the reactions of the wt, $\mathrm{Y} 68(3-\mathrm{FY})$, and $\mathrm{Y} 68\left(3,5-\mathrm{F}_{2} \mathrm{Y}\right)$ proteins, all at $350 \mathrm{~ms}$. $(\boldsymbol{C}, \boldsymbol{D})$ Spectra from the reactions of the wt protein at $350 \mathrm{~ms}$, the $\mathrm{Y} 68\left(4-\mathrm{NH}_{2} \mathrm{~F}\right)$ variant at $270 \mathrm{~ms}$, and the $\mathrm{Y} 68(3-\mathrm{ClY})$ variant at $420 \mathrm{~ms}$. (E, F) Spectra from the reactions of the wt protein at $350 \mathrm{~ms}$, the $\mathrm{Y} 224\left(4-\mathrm{NH}_{2} \mathrm{~F}\right)$ variant at $640 \mathrm{~ms}$, and the Y224(3-ClY) variant at $750 \mathrm{~ms}$. The reaction times for variants were 
selected to coincide with the time of maximal accumulation. The spectra from the wt FtmOx1 reaction are reproduced in each panel for ease of comparison. The absorption spectra have been scaled to coincide at the peak maxima, and the EPR spectra have been scaled to have equal double-integrated intensity; the scaling factors with respect to the wt spectra are noted in each panel. The absorption were directly extracted from the same SF-abs datasets used in preparing Figure S4, and the reaction conditions in the FQ-EPR experiments were the same as in the stopped-flow experiments (see Figure S4 caption).

To assess the possibility that Tyr224 can act as an alternative or secondary $\mathrm{H} \cdot$ donor (Figure S3), we evaluated variants with $4-\mathrm{NH}_{2} \mathrm{~F}$ or $3-\mathrm{ClY}$ in place of Tyr68 or Tyr224; substitution of either residue with these ncAAs was seen to preserve function (Figure 2). In the reaction of the $\mathrm{Y} 68\left(4-\mathrm{NH}_{2} \mathrm{~F}\right)$ variant, the radical accumulated maximally at $260 \mathrm{~ms}$ (Figure S5A) and absorbed at $\sim 409 \mathrm{~nm}$ (Figures 3C and S4E). This absorption maximum is consistent with that arising from the radical derived from 4methylaniline $^{36}$ (Figure 3C). The EPR spectrum of samples freeze-quenched near the time of maximum $409-\mathrm{nm}$ absorption has additional hyperfine splittings from both ${ }^{14} \mathrm{~N}(I$ $=1$ ) and the proton it harbors $(I=1 / 2)^{37}$, in contrast with the spinless, unprotonated ${ }^{16} \mathrm{O}$ in Tyros (Figure 3D). This observation is, to our knowledge, the first time that a simple anilino radical has been isolated and characterized in a protein. ${ }^{38}$ By contrast to the clear perturbation of the accumulating radical in the reaction of the $\mathrm{Y} 68\left(4-\mathrm{NH}_{2} \mathrm{~F}\right)$ variant, the Y224 $\left(4-\mathrm{NH}_{2} \mathrm{~F}\right)$ variant developed absorption (Figures 3E and S4I) and EPR spectra (Figure $3 F$ ) that matched exactly with their counterparts in the reaction of the wild-type enzyme [although this ncAA at position 224 did modestly delay maximal accumulation of the radical to $\sim 0.63 \mathrm{~s}$ (Figure $\mathrm{S} 5 \mathrm{C}$ )]. The same observations were made in examination of the $\mathrm{Y} 68(3-\mathrm{ClY})$ and $\mathrm{Y} 224(3-\mathrm{ClY})$ variant enzymes. The chlorinated tyrosine - expected to exhibit a narrower EPR spectrum when oxidized to a radical as a consequence of the diminished gyromagnetic ratios of ${ }^{35} \mathrm{Cl}$ and ${ }^{37} \mathrm{Cl}$ relative to ${ }^{1} \mathrm{H}^{34}-$ did indeed cause the expected narrowing when incorporated at position 68, but not when it was incorporated at position 224 (Figures 3C-F, S4D, and S4H). These observations with non-canonical variants thus definitively establish that Tyr68 rather than Tyr224 is the $\mathrm{H} \cdot$ donor in the FtmOx1 reaction, echoing the conclusions reached from investigation of the $\mathrm{Y} 68 \mathrm{~F}$ and $\mathrm{Y} 224 \mathrm{~F}$ variants ${ }^{10}$ and, more recently, from the structure of the FtmOx1•Fe(II)•2OG・1 complex (Figures S10A and S11). ${ }^{4}$ The new data are 
consistent with and rationalize the prior observation that the $\mathrm{Y} 224 \mathrm{~F}$ variant is fully competent to produce 2 but the Y68F generates an alternative primary product, identified in experiments discussed below.

Effect of the $\mathrm{H} \cdot-$ donor ability of residue 68 on the 2:5 partition ratio. With the ability more subtly to perturb the structure and properties of the position 68 residue now in hand, we sought to use this new tool to identify the alternative product, $\mathbf{5}$, of the Y68F variant (red trace in Figure $2 \mathrm{~A}$ ) and probe determinants of its formation. Two perturbations, in particular, were used in combination with mass spectrometry to identify the alternative product as 26-hydroxyverruculogen, resulting from a second $\mathrm{O}_{2}$ coupling step with the stabilized $\mathrm{C} 26 \cdot$. Incorporation of $2,3-\mathrm{F}_{2} \mathrm{Y}$ at position 68 was anticipated to slow $\mathrm{H} \cdot$ donation, because the $\mathrm{O}-\mathrm{H}$ bond of this Tyr analog is $\sim 1 \mathrm{kcal} / \mathrm{mol}$ stronger than that of tyrosine itself (Figure S2). Similarly, use of ${ }^{2} \mathrm{H}_{2} \mathrm{O}\left(\mathrm{D}_{2} \mathrm{O}\right)$ as solvent to replace the phenolic protium with deuterium was expected to slow the HAT step as a result of two effects: the increased strength of the O-D bond, which results primarily from its diminished vibrational zero-point energy, and the vastly diminished capacity of deuterium to undergo quantum-mechanical tunneling, which can contribute significantly to the efficiency of HAT reactions.

In SF-abs experiments with the $\mathrm{Y} 68\left(2,3-\mathrm{F}_{2} \mathrm{Y}\right)$ variant, we observed no transient absorption feature attributable to a Tyr (Figure S4K). Indeed, its kinetic behavior was seen to mirror that of the $\mathrm{Y} 68 \mathrm{~F}$ variant (Figures S5A and S5B). In principle, the diminished $p K_{a}$ of this analog ( 7.8 for the free amino acid) relative to that of tyrosine could cause it to deprotonate and lose competence for the quenching HAT step, but the full competence of 3,5- $\mathrm{F}_{2} \mathrm{Y}$ - with an even lower $\mathrm{p} K_{\mathrm{a}}(7.2)$ - at position 68 makes deprotonation an implausible explanation for the altered kinetic behavior of the Y68(2,3$\mathrm{F}_{2} \mathrm{Y}$ ) variant. Indeed, LC-MS analysis of the products from reactions of this variant revealed formation of primarily the native product, $\mathbf{2}$, but with a significant quantity of the alternative product, 5 (Figures $2 \mathrm{~A}$ and $\mathrm{S} 18$ ). Thus, the 2,3- $\mathrm{F}_{2} \mathrm{Y}$ analog is, unlike either tyrosine or phenylalanine, partly competent for the quenching HAT step. Presumably, the more potently oxidizing $2,3-\mathrm{F}_{2} \mathrm{Y} 68$ - has a diminished lifetime relative to that Tyr68 - and simply fails to accumulate under the reaction conditions examined. It is also 
possible that the diminished $\mathrm{p} K_{a}$ of this analog results in a fraction of enzyme bearing the HAT-incompetent phenolate. Irrespective of this detail, the toolkit of fluorinated analogs at position 68 thus afforded a variant bridging the fully competent wild-type enzyme and the incompetent $\mathrm{Y} 68 \mathrm{~F}$ variant, providing additional evidence that Tyr68 is the $\mathrm{H} \cdot$ donor.

A second strategy that we adopted to delay the quenching HAT step in order to assess if this delay leads to formation of $\mathbf{5}$ in competition with $\mathbf{2}$ was replacement of the phenolic hydrogen atom with deuterium in the wild-type enzyme. The substitution could be achieved simply by performing the assay in $\mathrm{D}_{2} \mathrm{O}$, owing to the fast ${ }^{1} \mathrm{H} /{ }^{2} \mathrm{H}$ exchange rate of solvent-exposed tyrosines $\left(>100 \mathrm{~s}^{-1}\right) .{ }^{27}$ Moreover, the much slower exchange of the newly formed C-D bond in 2 permitted deuterium substitution of the $\mathrm{H} \cdot$ donor to be verified by observation of a gain of one mass unit in the 2 produced in $\mathrm{D}_{2} \mathrm{O}$. Analysis of the products from the reaction of wild-type FtmOx1 in fully oxygenated $\mathrm{H}_{2} \mathrm{O}$ and $\mathrm{D}_{2} \mathrm{O}$ buffers (with $1 \mathrm{mM}$ ascorbate) revealed, as anticipated, a diminished formation of 2 in $\mathrm{D}_{2} \mathrm{O}$ (Figure 4A, compare blue and cyan traces). Consistent with earlier work, ${ }^{10}$ the yield of the native product was also much less in the reaction of the $\mathrm{Y} 68 \mathrm{~F}$ variant (red and orange traces), with the reaction of the $\mathrm{Y} 68 \mathrm{~F}$ variant in $\mathrm{D}_{2} \mathrm{O}$ producing almost no 2 . In all cases, the diminution in the yield of $\mathbf{2}$ was accompanied by an enhanced yield of $\mathbf{5}$ (see also Figure S18). The results with the $\mathrm{Y} 68\left(2,3-\mathrm{F}_{2} \mathrm{Y}\right)$ variant and wild-type FtmOx1 in $\mathrm{D}_{2} \mathrm{O}$ demonstrate that formation of $\mathbf{2}$ and $\mathbf{5}$ are competitive, with the former requiring the Tyr68-mediated HAT step and the latter proceeding via a different pathway not involving this step. 

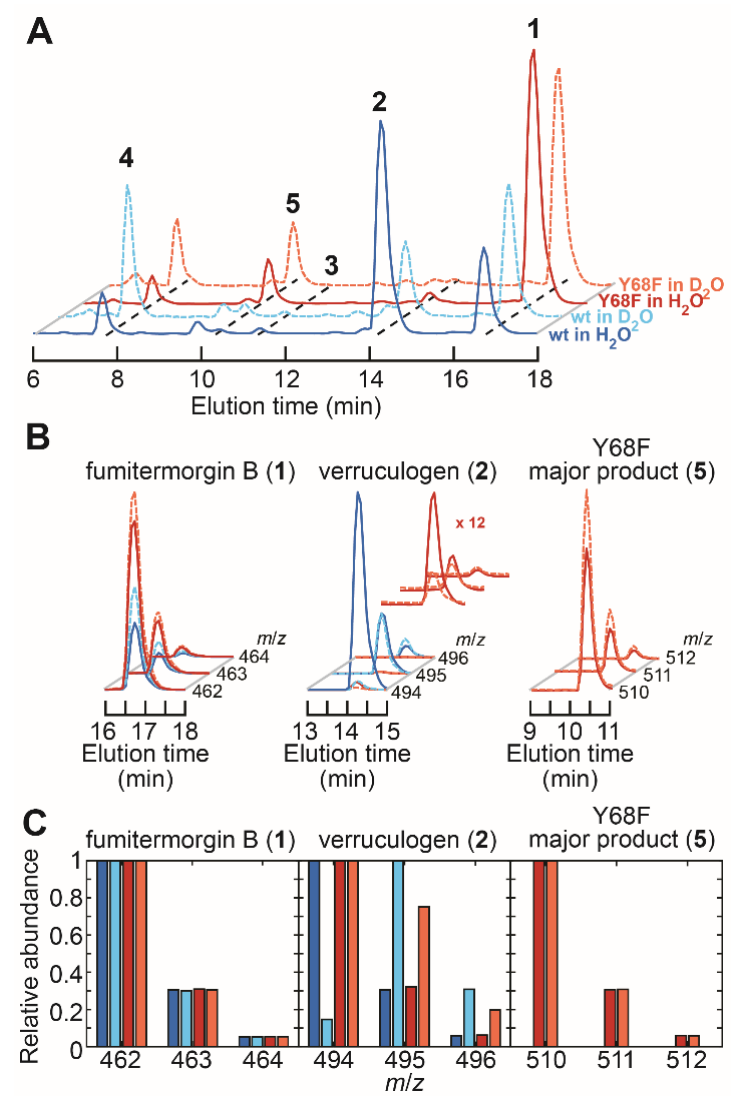

Figure 4. LC-MS characterization of products derived from 1 in reactions of wildtype Ftm Ox1 and its $\mathrm{Y} 68 \mathrm{~F}$ variant in $\mathrm{H}_{2} \mathrm{O}(\mathrm{pH} 8.0)$ and $\mathrm{D}_{2} \mathrm{O}(\mathrm{pD}$ 8.0). (A) Total-ion chromatograms (TICs) of products from the reactions of wt FtmOx1 in $\mathrm{H}_{2} \mathrm{O}$ (solid blue trace), wt enzymes in $\mathrm{D}_{2} \mathrm{O}$ (dashed cyan trace), the $\mathrm{Y} 68 \mathrm{~F}$ variant in $\mathrm{H}_{2} \mathrm{O}$ (solid red trace), and the $\mathrm{Y} 68 \mathrm{~F}$ variant in $\mathrm{D}_{2} \mathrm{O}$ (dashed orange trace). The color code also applies to panels $B$ and $C$. $(\boldsymbol{B})$ Isotope analysis from EICs of $\mathbf{1 , 2}$, and $\mathbf{5}$ formed in the reactions of panel $A$. Traces of $\mathbf{2}$ from the reaction of the Y68F variant are magnified to better illustrate the isotopic distributions. (C) Relative abundances of the isotopologues of $\mathbf{1}, \mathbf{2}$, and 5 extracted from panel $B$. The reactions contained $10 \mu \mathrm{M}$ enzyme, $10 \mu \mathrm{M} \mathrm{Fe}(\mathrm{II}), 1$ $\mathrm{mM} 2 \mathrm{OG}, 1 \mathrm{mM}$ ascorbate, $0.50 \mathrm{mM} \mathrm{1}$, and $~ 1.8 \mathrm{mM} \mathrm{O}_{2}, \mathrm{pH} / \mathrm{pD} 8.0$ at room temperature.

The observation in the prior study, ${ }^{10}$ confirmed by the present work (Figure 4A, red traces), that the FtmOx1 variant bearing the HAT-incompetent Phe at position 68 produces a detectable quantity of $\mathbf{2}$ implies that $\mathrm{H} \cdot$ can be provided (inefficiently) to C26 by one or more additional source when the preferred HAT donor is absent. Comparative mass-spectrometric analysis of 2 generated in $\mathrm{D}_{2} \mathrm{O}$ by the wild-type and $\mathrm{Y} 68 \mathrm{~F}$ proteins provided support for this conclusion. To verify the isotopic distribution of substrate $\mathbf{1}$ (which should be nearly identical to that of the product 2 formed in $\mathrm{H}_{2} \mathrm{O}$ ), the 
commercial compound was subjected to MS analysis and shown to conform to the predicted distribution $(m: m+1: m+2=1: 0.3: 0.05$; Figures 4B,C left), which arises primarily from the $1.1 \%$ natural abundance of ${ }^{13} \mathrm{C}$. A similar mass distribution was observed for 2 produced by either the wild-type or $\mathrm{Y} 68 \mathrm{~F}$ enzyme in $\mathrm{H}_{2} \mathrm{O}$ buffer $(m: m+1: m+2=1: 0.3: 0.06$; Figures 4B,C middle). By contrast, the mass spectrum observed for 2 produced by wild-type FtmOx1 in $\mathrm{D}_{2} \mathrm{O}$ buffer is dominated by the second lightest isotopologue $(m / z=495$ after dehydration; $m: m+1: m+2=1: 6.9: 2$ or $\sim 70 \%$; Figures 4B,C middle). This difference demonstrates that the $\mathrm{H} \cdot$ donated to $\mathrm{C} 26 \cdot$ is exchangeable with solvent, as would be expected for the phenolic hydrogen of a solvent accessible tyrosine such as Tyr68. The miniscule quantity of 2 produced by the Y68F variant in $\mathrm{D}_{2} \mathrm{O}$ buffer was also found to have more than the natural abundance of its $m+1$ isotopologue (Figures 4B,C middle), but, in this case, the heavier species was quantitatively much less prevalent (1:0.75:0.2, or $~ 38 \%)$ than in the corresponding reaction of the wild-type protein. The diminished deuterium incorporation in the reaction of the Y68F variant could originate from a less solvent-exposed hydrogen donor that incompletely exchanges with the solvent, a larger deuterium kinetic isotope effect $\left({ }^{2} \mathrm{H}\right.$ $\mathrm{KIE}$ ) that selects to a greater extent for incorporation of residual protium in the buffer (< $4 \%{ }^{1} \mathrm{H}$ ), or a combination of these effects. Regardless of the explanation, the differential deuterium incorporation implies that the Y68F variant utilizes a different $\mathrm{H} \cdot$ donor to generate 2 than does wild-type FtmOx1, again consistent with the assigned role of Tyr68.

\section{A third $\mathrm{O}_{2}$-derived oxygen atom in 5 suggests a second radical- $\mathrm{O}_{2}$ coupling.} Unexpectedly, we also discovered that the alternative product, 5, generated by either wild-type FtmOx1 or its $\mathrm{Y} 68 \mathrm{~F}$ variant in $\mathrm{D}_{2} \mathrm{O}$ buffer conforms to the natural isotopic abundance (Figures 4B,C right), indicating either that a deuterium is not incorporated in forming $\mathbf{5}$ or that any incorporated deuterium remains solvent exchangeable. In other words, no new $\mathrm{C}-\mathrm{H} / \mathrm{D}$ bond is formed in production of 5 . In assays with ascorbate, the 12,13-dihydroxy moiety is protected from further oxidation by Y68• (Figures S6 and S7), and its presence renders $\mathbf{5}$ also highly susceptible to dehydration during ionization, as previously found for both 1 and 2 . Positing that 5 , with a $\Delta(\mathrm{m} / \mathrm{z})=+48$ relative to 1 and $\Delta(m / z)=+16$ relative to 2 , might be 26-hydroxyverruculogen, we reasoned that this 
compound could form by coupling between the longer-lived (as a result of the absence of Tyr68 or presence of deuterium on the Tyr68 phenol) C26 and the hydroxo ligand (lower right state in Figure 1C), as proposed for Nvfl. ${ }^{8}$ However, the much greater separation of $\mathrm{C} 26$ from the cofactor seen in the recent $\mathrm{FtmOx} 1 \cdot \mathrm{Fe}(\mathrm{II}) \cdot 2 \mathrm{OG} \cdot 1$ structure (7.1 A) (Figure S12) than was previously observed between C3' of asnovolin A and the $\mathrm{Fe}(\mathrm{II})$ cofactor in the Nvfl structure (4.2 $\AA$ ) (Figure $\mathrm{S} 10 \mathrm{C}$ ) provides argument against this possibility and suggests an alternative pathway involving a COX-like capture of a second $\mathrm{O}_{2}$ equivalent by $\mathrm{C} 26$ - followed by reduction of the $\mathrm{C} 26$ peroxyl radical (C26-OO.) to the alcohol. This pathway would provide a rationale for the observation that the presence of ascorbate greatly augments formation of $\mathbf{5}$ in the reaction of the $\mathrm{Y} 68 \mathrm{~F}$ variant. ${ }^{10}$

To test this hypothesis for the identity of $\mathbf{5}$, we carried out enzymatic assays with ${ }^{18} \mathrm{O}_{2}$ substrate (Figure 5). To ensure minimal ${ }^{16} \mathrm{O}_{2}$ contamination during the assay, we also evaluated the isotopic composition of the resulting succinate, into which one Oatom from $\mathrm{O}_{2}$ is obligatorily incorporated, without the possibility of exchange (Figure 1A). ${ }^{39}$ The consistent $\sim 99 \%{ }^{18} \mathrm{O}$ incorporation efficiency for succinate (Figure S13) across all samples confirmed proper exclusion of atmosphere from the reactions. Product 2 generated by either wild-type FtmOx1 or the Y68F variant incorporated two ${ }^{18} \mathrm{O}$ atoms ( $81 \%$ and $69 \%$, respectively; Figures $5 \mathrm{~B}, \mathrm{C}$ middle), as anticipated from the endoperoxide bridge.6,40 A possible explanation for the modestly diminished incorporation efficiency is the unavoidable introduction of ambient ${ }^{16} \mathrm{O}_{2}$ during product extraction (see Materials and Methods), during which some product bearing endoperoxide with natural isotopic composition may form. By contrast, the corresponding analysis of $\mathbf{5}$ produced by the Y68F variant (Figures 5B,C right; see also Figure S14) shows that much more of this product bears three ${ }^{18} \mathrm{O}$ atoms (91\%) than bears two ${ }^{18} \mathrm{O}$ atoms $(\sim 1 \%)(m: m+2: m+4: m+6: m+8=0.08: 0009: 0.007: 1: 0.06)$. These observations support the assignment of $\mathbf{5}$ as 26 -hydroxyverruculogen and strongly favor a second $\mathrm{O}_{2}$-capture step over oxygen rebound as being responsible for incorporation of the third $\mathrm{O}_{2}$-derived oxygen. 

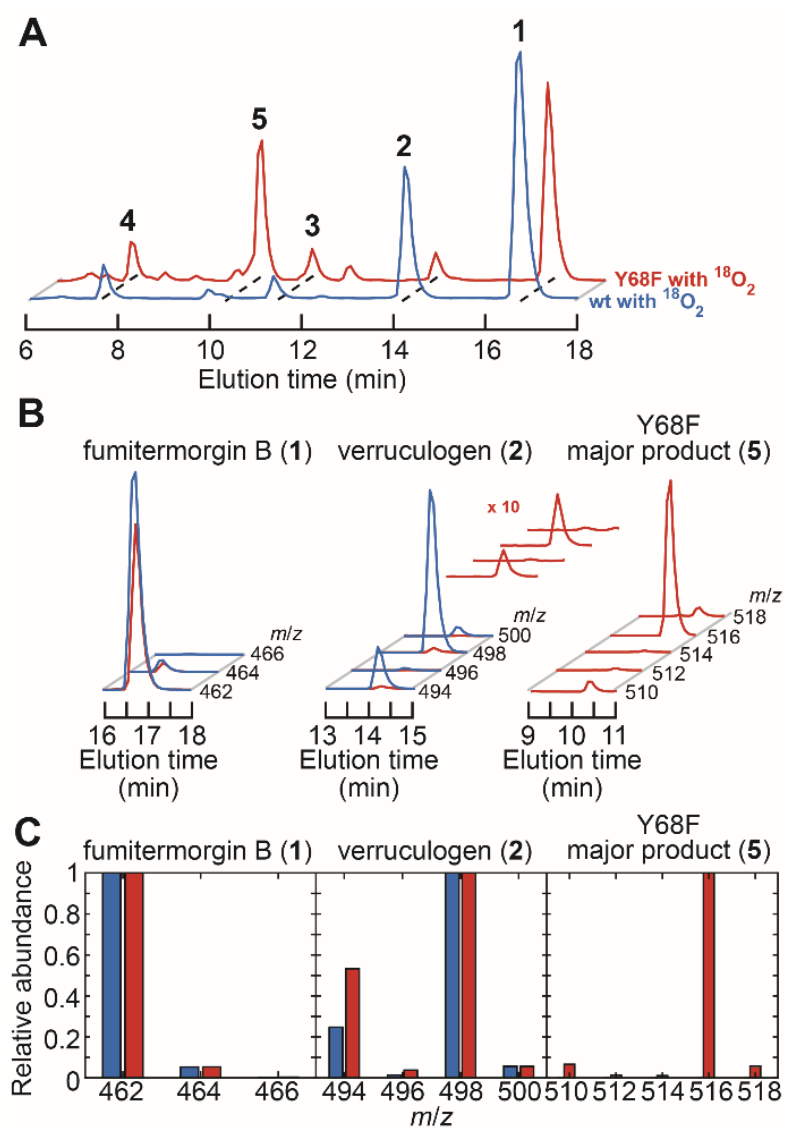

Figure 5. LC-MS characterization of products derived from 1 in reactions of wildtype FtmOx1 and its $\mathrm{Y} 68 \mathrm{~F}$ variant with ${ }^{18} \mathrm{O}_{2}$. $(A)$ TICs from the reaction of the wt (blue trace) and Y68F (red trace) enzymes. The same color code applies to panels $B$ and $C$. $(B)$ Isotope analysis from the EICs in $A$ of $\mathbf{1}, 2$, and 5 produced in these reactions. Traces for $\mathbf{2}$ from the Y68F variant are magnified to better illustrate the isotope distributions. $(\boldsymbol{C})$ Relative abundances for isotopologues of 1, 2, and 5 extracted from panel $B$. The reactions contained $510 \mu \mathrm{M}$ enzyme, $510 \mu \mathrm{M}$ Fe(II), $2.5 \mathrm{mM} 2 \mathrm{OG}, 250 \mu \mathrm{M}$ ascorbate, $0.25 \mathrm{mM} \mathrm{1}$, and $\sim 1.0 \mathrm{mM}^{18} \mathrm{O}_{2}, \mathrm{pH} 8.0$ at room temperature.

The commercial substrate 1 and its derived products $(2,3$, and 5$)$ formed with substrates and solvent of natural isotopic abundance were all further structural characterized by mass-spectrometric fragmentation analysis $\left(\mathrm{MS}^{2}\right)$. Even though the dominant ions were found to share the same nominal mass $(m / z=510)$ for $\mathbf{3}$ and $\mathbf{5}$, their fragmentation patterns are distinct (Figure S15). This observation, along with their different elution times, establish that they are different compounds. ${ }^{10}$ Interestingly, even though 2 and $\mathbf{5}$ were expected to differ by only one oxygen atom, their product ions could not be simply correlated, implying that they fragment quite differently. To facilitate fragment assignments, we utilized the isotopically labeled 2 and 5 produced in the 
reactions in $\mathrm{D}_{2} \mathrm{O}$ or with ${ }^{18} \mathrm{O}_{2}$ to ascertain whether certain atoms were retained (Figures $\mathrm{S} 16$ and S17). The assignments are listed in Table S1 and are consistent with a previous MS ${ }^{2}$ study of $2 .{ }^{41}$ The results suggest that, while 2 tends to retain at least one of the position 26 hydrogens (and C26 by association) upon fragmentation, as indicated by deuterium labeling, the spectra of 5 can be explained only by the presence of a heteroatom at C26 of 5 that promotes a-cleavage of the C3-C26 bond (numbering shown in Figure S17), causing C26 to be lost in the primary fragments generated. This pattern of fragmentation adds substantially to the evidence that 5 is 26hydroxyverruculogen.

\section{DISCUSSION}

Multiple roles and conformational heterogeneity of Tyr68. Through noncanonical substitutions of Tyr68 and Tyr224 of FtmOx1 and subsequent biophysical characterization, we can now unequivocally assign Tyr68 rather than Tyr224 as the radical-harboring site and the critical $\mathrm{H} \cdot$ donor, supporting the CarC-like mechanism (Figure 1C). The recently published structure of the $\mathrm{FtmOx} 1 \cdot \mathrm{Fe}(\mathrm{II}) \cdot 2 \mathrm{OG} \cdot 1$ quaternary complex reveals sufficient proximity (4.6 $\AA$ ) between $\mathrm{Fe}(\mathrm{II})$ and $\mathrm{C} 21$ to enable direct hydrogen abstraction by the ferryl moiety (Figures S10A). ${ }^{4}$ This structure implies that, associated with binding of 1, Tyr224 unexpectedly twists away from Fe(II) (Figure S1). Its location in the $\mathrm{O}_{2}$-reactive complex strongly disfavors its participation as initial $\mathrm{H} \cdot$ donor to the ferryl complex in a COX-like mechanism. By contrast, as in CarC, ${ }^{11}$ the loop on which the actual $\mathrm{H} \cdot$ donor resides (Tyr68 in FtmOx1 and Tyr165 in CarC) becomes less disordered (partially due to crystal packing, vide infra) and moves toward the active site upon substrate binding (Figure S1). The short distance $(4.0 \AA$ ) between the oxygen of Tyr68 and C26 of 1 would be expected to enable the terminating HAT step of the proposed CarC-like mechanism (Figures S10A and S11). The offline arrangements of $2 \mathrm{OG}$ and the $\mathrm{Fe}(\mathrm{II})$-bound water in the active site of FtmOx1 are also reminiscent of those seen in the structure of the CarC quaternary complex (Figures S10A and S10B). 
Intriguingly, another short distance ( $3.7 \AA$ ) between the phenolic oxygen of Tyr68 and $\mathrm{C} 13$ of 1 (Figures $\mathrm{S} 10 \mathrm{~A}$ and $\mathrm{S} 11$ ) is observed and is consistent with ensuing oxidation of $\mathbf{2}$ to 3 by the Tyr68 intermediate (Figures S7 and S8). It seems likely that decay of Tyr68 proceeds, at least in part, through HAT from $\mathrm{C}_{1} 3^{4}$ to generate a ketyl radical on the pathway to the formation of $\mathbf{3}$. However, given that $\mathbf{2}$ is not quantitatively oxidized during decay of Tyr68 (Figures S7 and S8), pathways other than that initiated by HAT from $\mathrm{C} 13$ must exist. Further inspection of the $\mathrm{FtmOx} 1 \cdot \mathrm{Fe}(\mathrm{II}) \cdot 2 \mathrm{OG} \cdot 1$ structure suggests that, even though the electron density implies a single Tyr68 conformation, in which the phenol stacks upon the indole ring of $\mathbf{1}$, the conformation of the segment on which Tyr68 resides is artificially restricted by neighboring symmetry-related monomers unique to the crystalline environment (Figure S21). By contrast, in the structure of the FtmOx1•Fe(II)·2OG ternary complex, the surface loop harboring Tyr68 is more disordered (Figure S20). Quantum mechanical simulations from the quaternary complex structure also suggest conformational flexibility of Tyr68 $\cdot{ }^{4}$ Indeed, if Tyr68 were to assume a single conformation in the apparently optimal position for HAT to $\mathrm{C} 13$, as the crystal structure suggests (Figures S10A, S11, S21A, and S21B), further oxidation of 2 by Tyr68 - would not be expected to proceed as slowly as the Tyr68 is observed to decay $\left(0.4 \mathrm{~s}^{-1}\right.$; Table S2), and the competition by other decay pathways, such as the implied reduction by ascorbate, would seem unlikely. One might even expect HAT from C13 of $\mathbf{1}$ to Tyr68 to be sufficiently rapid to prevent accumulation of Tyr68 during a single turnover. In this regard, as a solvent-exposed redox-active residue in FtmOx1, Tyr68 brings the remaining oxidizing equivalent to the protein surface after product formation, where the resulting radical can readily be reductively quenched. ${ }^{42}$ This situation is reminiscent of cofactor assembly in the radical generating subunit $(\beta)$ of the class la ribonucleotide reductase, in which a near-surface and conserved tryptophan (W48 in the E. coli homolog) plays a similar role. ${ }^{43}$

The proposed conformational heterogeneity of Tyr68 • is consistent with its broad and poorly resolved EPR signature (Figures 3 and S9), which does not coincide with spectra predicted for a Tyr ${ }^{\circ}$ with any single dihedral angle ${ }^{44}$ but rather resembles the spectra of free tyrosyl radicals in frozen glasses, in which conformational heterogeneity is expected. ${ }^{33}$ This situation contrasts with that in CarC, in which the unusually broad 
EPR lineshape of Tyr165 observed at $10 \mathrm{~K}$ was attributed to efficient relaxation by dipolar coupling to the paramagnetic Fe(III) center, ${ }^{11}$ an assignment confirmed by the observation of the underlying hyperfine structure - which matched that predicted according to the dihedral angle of Tyr165 modeled in the crystal structure (Figure $\mathrm{S} 10 \mathrm{~B})$ - when the EPR spectrum was measured at $80 \mathrm{~K}$. Indeed, acquisition of spectra of Tyr68 in FtmOx1 at higher temperatures led only to general broadening; it did not reveal the defined hyperfine couplings to the $\mathrm{C} 3$ proton(s) expected of a single welldefined side-chain conformation (Figure S22). This disparate behavior of the radicals in CarC and FtmOx1 supports the notion that conformational heterogeneity is the dominant cause of the unresolved hyperfine coupling in the spectrum of Y68•, which is consistent with the longer $\mathrm{Fe}(\mathrm{II})$-to-phenolic-oxygen distance in FtmOx1 $(10.6 \AA)^{4}$ than in CarC $(8.6 \AA)^{11}$ seen in crystal structures. This heterogeneity could also be responsible for the broad UV-vis absorption band of Tyr68. It has been reported that the UV-vis absorption of a Tyr• in a cryptochrome is red-shifted $(417 \mathrm{~nm})$ due to $\pi-\pi$ stacking with a nearby tryptophan, ${ }^{45}$ reminiscent of the interaction between Tyr68 and 1 seen in the recent crystal structure. ${ }^{4}$ Heterogeneity in the conformation of Tyr68 would render $\pi-\pi$ stacking less effective in some fraction of the population and thus broaden the absorption peak. The narrowing of both the UV-vis and EPR features of the 3CIY68- compared to its unsubstituted counterpart could be attributed to a restriction in conformation due to the steric bulk of chlorine (Figures 3C and 3D).

Tyr224- as the second radical after one catalytic cycle. It was suspected on the basis of kinetic results for the Y224F variant of FtmOx1 that Tyr224 could harbor a radical later in the reaction sequence, after verruculogen production is completed by HAT from Tyr68. ${ }^{10}$ This radical consistently exhibited a more usual absorption maximum at $409 \mathrm{~nm}$ in the wild-type enzyme and variants that permitted its accumulation (Figure S4), including the Y68F variant. For the Y224(3-CIY) variant, however, an absorption maximum of $404 \mathrm{~nm}$ was seen in the later-accumulating species (Figures $\mathrm{S} 4 \mathrm{H}$ and S5D), suggesting that Tyr224 could indeed harbor the second radical detected in the prior work. ${ }^{10}$ This assignment could imply another pathway by which FtmOx1 manages its remaining oxidizing equivalent (the Tyr68•) in order to avoid deleterious effects. ${ }^{42}$ Alternatively, the long reaction time at which the second Tyr• develops could reflect its 
formation in a second $\mathrm{O}_{2}$-activation event (e.g., triggered by product 3 ) that proceeds through a different pathway and leads to oxidation of Tyr224 rather than Tyr68. ${ }^{3,46}$

Hydrogen donation from Tyr68 determines the fate of the substrate radical. By interrogation of the reactions of wild-type FtmOx1 in $\mathrm{D}_{2} \mathrm{O}$ and the $\mathrm{Y} 68\left(2,3-\mathrm{F}_{2} \mathrm{Y}\right)$ variant, we showed that production of 5 and 2 compete, with the production of the alternative product $\mathbf{5}$ not involving the HAT step. The demonstrated incorporation of three $\mathrm{O}_{2}$-derived oxygen atoms into $\mathbf{5}$ is a telling clue as to the mechanism of its formation. It has been demonstrated that the ${ }^{18} \mathrm{O}$-labeled hydroxo ligand to $\mathrm{Fe}$ (III) formed after the $\mathrm{C}-\mathrm{H}$ cleavage step can be, in some Fe/2OG enzymes, susceptible to solvent exchange. ${ }^{39}$ Given the long-lived nature of the hydroxo- $\mathrm{Fe}(\mathrm{III})$ state in the FtmOx1 reaction (a lifetime of subseconds to seconds in the wild-type enzyme ${ }^{3}$ ), it is expected that the hydroxo group should have undergone significant solvent exchange in the cases that the formation of the alternative product competes effectively with formation of 2 by HAT to the C26 intermediate. Consequently, even if the substrate radical were ultimately resolved by oxygen rebound, 5 would not be expected to harbor three $\mathrm{O}_{2}$-derived oxygen atoms in its predominant isotopologue. The more likely pathway to $\mathbf{5}$ is for $\mathrm{C} 26$ to capture another $\mathrm{O}_{2}$ molecule when the HAT step is slowed (red arrow in Figure 6), as in the cases of both prostaglandin $\mathrm{G}_{2}$ formation catalyzed by $\mathrm{COX}^{2}$ and non-enzymatic free radical lipid peroxidation. ${ }^{47}$ In this most probable pathway, the resulting peroxyl radical would then be reduced by ascorbate to yield the C26 hydroxyl of 5 .

It is also informative that, for the reactions of both wild-type FtmOx1 and its Y68F variant, more 4, which is produced by oxygen rebound (cyan arrow in Figure 6) and subsequent non-enzymatic elimination (Figure $1 A$ ), is generated in $\mathrm{D}_{2} \mathrm{O}$ than in $\mathrm{H}_{2} \mathrm{O}$ (Figure 4A). This observation implies that each step between the HATs from C21 to the ferryl complex and from Tyr68 to the C26 - must be readily reversible (right side of Figure 1C; Figure 6), highlighting the importance of an efficient HAT step to complete the reaction, irreversibly fix the endoperoxide moiety, and avert oxygen rebound. The reversibility of $\mathrm{O}_{2}$ addition to and dissociation from allylic radicals has been noted in the literature on lipid peroxidation, ${ }^{47}$ inferred from interconversion of thermodynamically and 
kinetically controlled products. The secondary aliphatic C26 - formed upon olefinic addition of the peroxyl radical is presumably less thermodynamically favored than the allylic C21•, such that even a modestly slowed HAT step (orange arrow in Figure 6 ) could be enough to favor the rebound product. However, the capture of another $\mathrm{O}_{2}$ equivalent by $\mathbf{C 2 6}$ (yielding 5, red arrow in Figure 6) competes with the reversion to the rebound-susceptible C21 precursor to 4 (cyan arrows in Figure 6). The efficiency of C26• $\leftrightarrow \mathrm{O}_{2}$ coupling explains why a modest quantity of $\mathbf{5}$ is produced in reactions in which HAT to C26 is only subtly impeded, such as in the reaction of either wild-type FtmOx1 in $\mathrm{D}_{2} \mathrm{O}$ (Figure $4 \mathrm{~A}$ ) or the $\mathrm{Y} 68\left(2,3-\mathrm{F}_{2} \mathrm{Y}\right.$ ) variant in $\mathrm{H}_{2} \mathrm{O}$ (Figure $2 \mathrm{~A}$, see also Figure S18 for $\mathrm{MS}^{2}$ analysis of $\mathbf{5}$ from these sources). It is curious, then, that the perturbation expected to most profoundly impede HAT to C26 - namely, the Y68F substitution - overwhelmingly favors production of $\mathbf{5}$ over $\mathbf{4}$. As Tyr68 appears ideally poised in the wild-type quaternary complex ${ }^{4}$ to donate $\mathrm{H} \cdot$ to $\mathrm{C} 26 \cdot$ in the intermediate state, the preferred outcome of the $\mathrm{Y} 68 \mathrm{~F}$ variant might be attributable to an enlarged protein pocket favoring access of $\mathrm{O}_{2}$ to the $\mathrm{C} 26 \cdot$ intermediate (Figure S12). The more structurally conservative nature of the more modestly HAT-impeding perturbations (solvent deuteration and the non-canonical tyrosine) do not have this secondary impact on $\mathrm{O}_{2}$ access to the key product radical, making formation of 5 less favored.

It thus appears that the multiple substrate radical species on the FtmOx1catalyzed pathway to $\mathbf{2}$ conform to the behavior that chemically similar species have been found to exhibit in non-enzymatic contexts. ${ }^{47}$ It is the presence of Tyr68 and its ability to efficiently donate $\mathrm{H} \bullet$ to the $\mathrm{C} 26 \bullet$ intermediate that engenders selectivity for production of $\mathbf{2}$ over formation of $\mathbf{4}$ or $\mathbf{5}$ by oxygen rebound or C26 $\leftrightarrow \mathrm{O}_{2}$ coupling, respectively. To frame this scheme quantitatively, we can assign a rate constant (or an equilibrium constant) to each step in the progression from 1 to 2 (Figure 6). For 2 to predominate in the reaction of wild-type FtmOx1, two branch points, corresponding to the radical residing on $\mathrm{C} 21$ and $\mathrm{C} 26$, must be navigated. Successful navigation requires, first, that the effective first-order rate constant for $\mathrm{O}_{2}$ addition to $\mathrm{C} 21 \cdot\left(k_{1}\left[\mathrm{O}_{2}\right]\right)$ be greater than the first-order rate constant for oxygen rebound krebound. The second-order rate constant for $\mathrm{O}_{2}$ addition to allylic radicals, corresponding to $k_{1}$, has been determined to be $\sim 10^{9} \mathrm{M}^{-1} \mathrm{~s}^{-1}$ in non-enzymatic systems, ${ }^{47}$ and so one expects $\mathrm{O}_{2}$ addition to $\mathrm{C} 21 \cdot$ to 
occur on the microsecond timescale in our experiments $\left(\left[\mathrm{O}_{2}\right] \sim 1 \mathrm{mM}\right)$. By comparison, oxygen rebound can be very fast $\left(10^{10}-10^{11} \mathrm{~s}^{-1}\right)$ in heme-dependent hydroxylases. ${ }^{48,49}$ For wild-type FtmOx1, the rebound step competes effectively and even dominates under low $\left[\mathrm{O}_{2}\right],{ }^{10}$ which might seem to suggest only modest proficiency in control. However, to be able even to form 2, krebound must be significantly slowed - by a mechanism that is not yet known - relative to the aforementioned heme-dependent hydroxylases. Second, HAT to $\mathrm{C} 26$ • has to be faster than the second $\mathrm{O}_{2}$ capture and ring dissociation, which are also estimated to have rate constants of $\sim 10^{6} \mathrm{~s}^{-1}$ from studies of non-enzymatic lipid peroxidation. ${ }^{47}$ The same competition between HAT (usually by a-tocopherol, another phenol-based hydrogen donor) and further $\mathrm{O}_{2}$ capture has also been interrogated in lipid peroxidation. ${ }^{2,47,50}$ It would not be surprising if $k_{\text {HAT }}$ in FtmOx1 is of similar order of magnitude, given that formation of 4 by rebound is drastically favored in $\mathrm{D}_{2} \mathrm{O}$. Unfortunately, direct extraction of $k_{\mathrm{HAT}}$ from Figure $\mathrm{S} 5$ is not possible with the existing data. The fast equilibria between $\mathrm{C} 21 \cdot$, the peroxyl radical, and C26• are also estimated to occur on the microsecond timescale, ${ }^{50}$ which are well within the deadtime of our stopped-flow experiments, and so it is not surprising that the

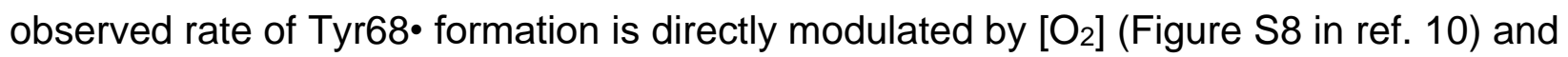
is convolved with all the prior elementary steps before HAT $\left(\mathrm{O}_{2}\right.$ addition to the cofactor, $20 \mathrm{G}$ decarboxylation, etc.). Studying the product distribution (equation in Figure 6 ) as a function of $\left[\mathrm{O}_{2}\right]$ might provide a means of estimating $\mathrm{KHAT}_{\mathrm{HA}}$, or at least of extracting trends thereupon, in the reactions of variants bearing non-canonical tyrosine analogs. 


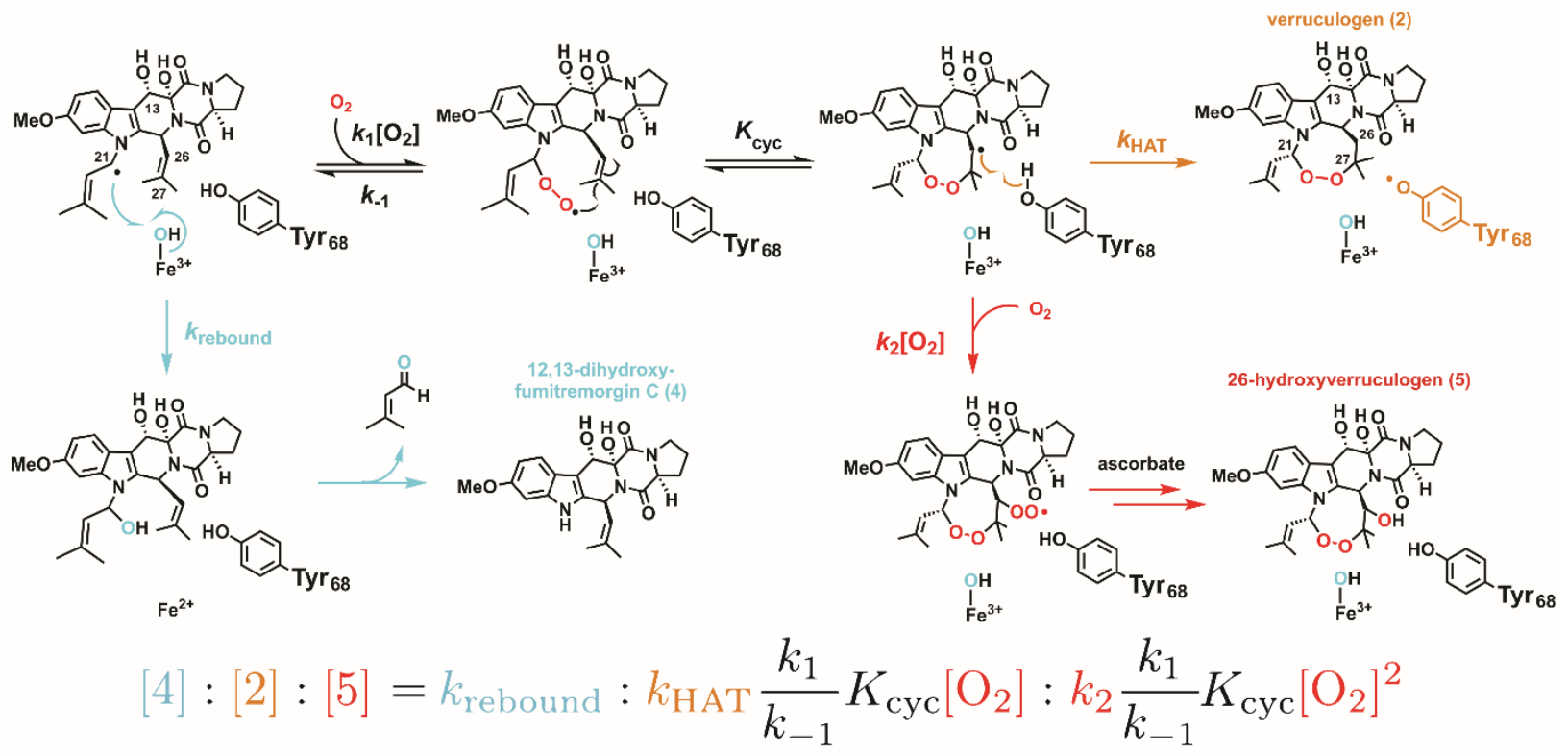

Figure 6. Origins of the dominant 1-derived products (except for 3 , which is shown in Figure $1 \mathrm{C}$ ) and the fate of the substrate radical. The rate constants and equilibrium constant are labeled at each critical step, and the predicted product ratio is governed by the oxygen concentration $\left[\mathrm{O}_{2}\right]$, the rebound rate constant ( $\left.k_{\text {rebound }}\right)$, the HAT rate constant ( $k_{\text {HAT }}$ ), and various rate constants for oxygen addition, dissociation, and cyclization. The equation assumes rapid equilibria among the radical intermediates (see text).

\section{CONCLUSIONS}

With the aid of non-canonical tyrosine analog incorporation at the controversial sites 68 and 224 in FtmOx1, we were able to unambiguously locate the transient radical formed from Tyr68 and exclude the possibility of a COX-like mechanism. Isotope labeling studies in combination with the Y68F substitution mapped out the possible fates of the substrate radical intermediates produced subsequent to HAT from C21 (Figure 6), elucidating how FtmOx1 partially suppresses rebound even in the absence of the key $\mathrm{H} \cdot-$ donating Tyr68. The fact that subtly perturbing Tyr68 through halogenation and/or deuteration is enough to alter the partition ratio of ftnB-derived products suggests that rate constants governing the fates of branch-point intermediates are similar. The role of Tyr68 and Tyr68 in verruculogen formation and oxidation, respectively, were established through chemical-quench studies. The emergent mechanism resembles 
that of CarC even more closely than originally recognized, in the sense that a critical tyrosine donates $\mathrm{H} \cdot$ to prevent hydroxylation by oxygen rebound. To our knowledge, this study represents the first systematic deployment of non-canonical tyrosine analogs to study mechanisms and control of HAT reactions in proteins; it thus complements prior studies that have used either the same approach on proton-coupled electron transfers in proteins ${ }^{17,26,30-35}$ or analogous substituted phenols to probe HAT reactions in solution. ${ }^{47,50,51}$ In addition, this study reinforces the importance of accurately visualizing the position of the prime substrate in the authentic $\mathrm{O}_{2}$-reactive complex in attempts to understand the chemical mechanism of an Fe/2OG enzyme.

As rare and exotic as they may appear, endoperoxides are pervasive products from allylic systems, such as unsaturated lipids, through non-enzymatic radical peroxidation. ${ }^{47}$ The enzyme environments, including those of COX and FtmOx1, take advantage of this chemistry but direct the reaction outcomes through precise positioning of hydrogen donors with appropriate BDEs to avoid both oxygen rebound and undesired radical $\leftrightarrow \mathrm{O}_{2}$ couplings. It is likely that more endoperoxidases that produce bioactive secondary metabolites through variant mechanisms await discovery. For instance, while both $\mathrm{Fe} / 2 \mathrm{OG}$ endoperoxidases catalyze endoperoxide bridge installation followed by hydroxylation, Nvfl executes the hydroxylation step via oxygen rebound, ${ }^{7}$ whereas the Y68F variant of FtmOx1 instead utilizes a second $\mathrm{O}_{2}$ capture. FtmOx1 (and its variants) could be an excellent model system for interrogating endoperoxidation, providing guidance in the mechanistic dissection of yet unknown endoperoxidases.

\section{ASSOCIATED CONTENT}

\section{Supporting Information}

Structural analyses of FtmOx1 and related Fe/2OG oxygenases, stopped-flow traces and kinetic analyses, chemical-quench results, 2OG binding analysis for variants, further EPR and MS data, NMR spectra for fluorotyrosines, SDS-PAGE analysis and ESI-MS spectra for variants, and assignments of $\mathrm{MS}^{2}$ product fragments.

\section{ACKNOWLEDGMENTS}


We thank Dr. Noah Dunham for helpful discussions. C.-Y.L. was supported by a grant from The Jane Coffin Childs Memorial Fund for Medical Research. This work was supported. by grants from the National Institutes of Health (GM113106 to J.M.B./C.K., GM127079 to C.K., and GM119707 to A.K.B.). 


\section{REFERENCES}

(1) Liu, D.-Z.; Liu, J.-K. Peroxy natural products. Nat. Prod. Bioprospect 2013, 3, 161206.

(2) Tsai, A.-L.; Kulmacz, R. J. Prostaglandin H synthase: Resolved and unresolved mechanistic issues. Arch. Biochem. Biophys. 2010, 493, 103-124.

(3) Yan, P.; Song, H.; Song, F.; Guo, Y.; Wu, C.-H.; Her, A. S.; Pu, Y.; Wang, S.; Naowarojna, N.; Weitz, A.; Hendrich, M. P.; Costello, C. E.; Zhang, L.; Liu, P.; Zhang, Y. S. Endoperoxide formation by an a-ketoglutarate-dependent mononuclear non-haem iron enzyme. Nature 2015, 527, 539-543.

(4) Wu, L.; Wang, Z.; Cen, Y.; Wang, B.; Zhou, J. Structural insight into the catalytic mechanism of endoperoxide synthase FtmOx1. Angew. Chem Int. Ed. 2022 DOI: 10.1002/anie.202112063.

(5) Rouzer, C. A.; Marnett, L. J. Structural and chemical biology of the interaction of cyclooxygenase with substrates and non-steroidal anti-inflammatory drugs. Chem. Rev. 2020, 120, 7592-7641.

(6) Steffan, N.; Grundmann, A.; Afiyatullov, S.; Ruan, H.; Li, S-.M. FtmOx1, a nonheme $\mathrm{Fe}(\mathrm{II})$ and a-ketoglutarate-dependent dioxygenase, catalyses the endoperoxide formation of verruculogen in Aspergillus fumigatus. Org. Biomol. Chem. 2009, 7, 4082-4087.

(7) Matsuda, Y.; Bai, T.; Phippen, C. B. W.; Nødvig, C. S.; Kjærbølling, I.; Vesth, T. C.; Andersen, M. R.; Mortensen, U. H.; Gotfredsen, C. H.; Abe, I.; Larsen, T. O. Novofumigatonin biosynthesis involves a non-heme iron-dependent endoperoxide isomerase for orthoester formation. Nat. Commun. 2018, 9, 2587.

(8) Mori, T.; Zhai, R.; Ushimaru, R.; Matsuda, Y.; Abe, I. Molecular insights into the endoperoxide formation by Fe(II)/a-KG-dependent oxygenase Nvfl. Nat. Commun. 2021, 12, 4417.

(9) Bollinger, J. M., Jr.; Chang, W.-c.; Matthews, M. L.; Martinie, R. J.; Boal, A. K.; Krebs, C. Mechanisms of 2-Oxoglutarate-Dependent Oxygenases: The Hydroxylation Paradigm and Beyond. In 2-Oxoglutarate-Dependent Oxygenases; Hausinger, R. P., Schofield, C. J., Eds.; The Royal Society of Chemistry: London, 2015; pp 95-122. 
(10) Dunham, N. P.; Del Río Pantoja, J. M.; Zhang, B.; Rajakovich, L. J.; Allen, B. D.; Krebs, C.; Boal, A. K.; Bollinger, J. M., Jr. Hydrogen donation but not abstraction by a tyrosine (Y68) during endoperoxide installation by verruculogen synthase (FtmOx1). J. Am. Chem. Soc. 2019, 141, 9964-9979.

(11) Chang, W.-c.; Guo, Y.; Wang, C.; Butch, S. E.; Rosenzweig, A. C.; Boal, A. K.; Krebs, C.; Bollinger, J. M., Jr. Mechanism of the C5 stereoinversion reaction in the biosynthesis of carbapenem antibiotics. Science 2014, 343, 1140-1144.

(12) Groves, J. T. Key elements of the chemistry of cytochrome P-450: The oxygen rebound mechanism. J. Chem. Educ. 1985, 62, 928-931.

(13) Yan, P.; Song, H.; Song, F.; Guo, Y.; Wu, C.-H.; Her, A. S.; Pu, Y.; Wang, S.; Naowarojna, N.; Weitz, A.; Hendrich, M. P.; Costello, C. E.; Zhang, L.; Liu, P.; Zhang, Y. S. Retraction note: Endoperoxide formation by an a-ketoglutaratedependent mononuclear non-haem iron enzyme. Nature 2021, 593, 612.

(14) Naowarojna, N.; Cheng, R.; Lopez, J.; Wong, C.; Qiao, L.; Liu, P. Chemical modifications of proteins and their applications in metalloenzyme studies. Synth. Syst. Biotechnol. 2021, 6, 32-49.

(15) Young, D. D.; Schultz, P. G. Playing with the molecules of life. ACS Chem. Biol. 2018, 13, 854-870.

(16) Hirel, P.-H.; Schmitter, J.-M.; Dessen, P.; Fayat, G.; Blanquet, S. Extent of Nterminal methionine excision from Escherichia coli proteins is governed by the side-chain length of the penultimate amino acid. Proc. Natl. Acad. Sci. U. S. A. 1989, 86, 8247-8251.

(17) Minnihan, E. C.; Young, D. D.; Schultz, P. G.; Stubbe, J. Incorporation of fluorotyrosines into ribonucleotide reductase using an evolved, polyspecific aminoacyl-tRNA synthetase. J. Am. Chem. Soc. 2011, 133, 15942-15945.

(18) Liu, X.; Jiang, L.; Li, J.; Wang, L.; Yu, Y.; Zhou, Q.; Lv, X.; Gong, W.; Lu, Y.; Wang, J. Significant expansion of fluorescent protein sensing ability through the genetic incorporation of superior photo-induced electron-transfer quenchers. J. Am. Chem. Soc. 2014, 136, 13094-13097. 
(19) Mehl, R. A.; Anderson, J. C.; Santoro, S. W.; Wang, L.; Martin, A. B.; King, D. S.; Horn, D. M.; Schultz, P. G. Generation of a bacterium with a 21 amino acid genetic code. J. Am. Chem. Soc. 2003, 125, 935-939.

(20) Young, D. D.; Young, T. S.; Jahnz, M.; Ahmad, I.; Spraggon, G.; Schultz, P. G. An evolved aminoacyl-tRNA synthetase with atypical polysubstrate specificity. Biochemistry 2011, 50, 1894-1900.

(21) Drienovská, I.; Mayer, C.; Dulson, C.; Roelfes, G. A designer enzyme for hydrazone and oxime formation featuring an unnatural catalytic aniline residue. Nat. Chem. 2018, 10, 946-952.

(22) Romei, M. G.; Lin, C.-Y.; Mathews, I. I.; Boxer, S. G. Electrostatic control of photoisomerization pathways in proteins. Science 2020, 367, 76-79.

(23) Gil, A. A.; Haigney, A.; Laptenok, S. P.; Brust, R.; Lukacs, A.; Iuliano, J. N.; Jeng, J.; Melief, E. H.; Zhao, R.-K.; Yoon, E.; Clark, I. P.; Towrie, M.; Greetham, G. M.; Ng, A.; Truglio, J. J.; French, J. B.; Meech, S. R.; Tonge, P. J. Mechanism of the AppAвLUF photocycle probed by site-specific incorporation of fluorotyrosine residues: Effect of the $\mathrm{Y} 21 \mathrm{p} K \mathrm{a}$ on the forward and reverse ground-state reactions. J. Am. Chem. Soc. 2016, 138, 926-935.

(24) Gill, S. C.; von Hippel, P. H. Calculation of protein extinction coefficients from amino acid sequence data. Anal. Biochem. 1989, 182, 319-326.

(25) Winkler, R. ESIprot: A universal tool for charge state determination and molecular weight calculation of proteins from electrospray ionization mass spectrometry data. Rapid Commun. Mass Spectrom. 2010, 24, 285-294.

(26) Olshansky, L.; Stubbe, J.; Nocera, D. G. Charge-transfer dynamics at the $\alpha / \beta$ subunit interface of a photochemical ribonucleotide reductase. J. Am. Chem. Soc. 2016, 138, 1196-1205.

(27) Takeda, M.; Jee, J.; Ono, A. M.; Terauchi, T.; Kainosho, M. Hydrogen exchange rate of tyrosine hydroxyl groups in proteins as studied by the deuterium isotope effect on $\mathrm{C}_{\zeta}$ chemical shifts. J. Am. Chem. Soc. 2009, 131, 18556-18562.

(28) Copeland, R. A.; Davis, K. M.; Shoda, T. K. C.; Blaesi, E. J.; Boal, A. K.; Krebs, C.; Bollinger, J. M., Jr. An iron(IV)-oxo intermediate initiating L-arginine oxidation but 
not ethylene production by the 2-oxoglutarate-dependent oxygenase, ethyleneforming enzyme. J. Am. Chem. Soc. 2021, 143, 2293-2303.

(29) Martinie, R. J.; Livada, J.; Chang, W.-c.; Green, M. T.; Krebs, C.; Bollinger, J. M., Jr.; Silakov, A. Experimental correlation of substrate position with reaction outcome in the aliphatic halogenase, SyrB2. J. Am. Chem. Soc. 2015, 137, 69126919.

(30) Oyala, P. H.; Ravichandran, K. R.; Funk, M. A.; Stucky, P. A.; Stich, T. A.; Drennan, C. L.; Britt, R. D.; Stubbe, J. Biophysical characterization of fluorotyrosines probes site-specifically incorporated into enzymes: $E$. coli ribonucleotide reductase as an example. J. Am. Chem. Soc. 2016, 138, 79517964.

(31) Rappaport, F.; Boussac, A.; Force, D. A.; Peloquin, J.; Brynda, M.; Sugiura, M.; Un, S.; Britt, R. D.; Diner, B. A. Probing the coupling between proton and electron transfer in photosystem II core complexes containing a 3-fluorotyrosine. J. Am. Chem. Soc. 2009, 131, 4425-4433.

(32) Yokoyama, K.; Smith, A. A.; Corzilius, B.; Griffin, R. G.; Stubbe, J. Equilibration of tyrosyl radicals $\left(\mathrm{Y}_{356^{\circ}}, \mathrm{Y}_{731^{\circ}}, \mathrm{Y}_{730^{\circ}}\right)$ in the radical propagation pathway of the Escherichia coli class la ribonucleotide. J. Am. Chem. Soc. 2011, 133, 1842018432.

(33) Seyedsayamdost, M. R.; Reece, S. Y.; Nocera, D. G.; Stubbe, J. Mono-, di-, tri-, and tetra-susbstitued fluorotyrosines: New probes for enzymes that use tyrosyl radicals in catalysis. J. Am. Chem. Soc. 2006, 128, 1569-1579.

(34) Yu, Y.; Lv, X.; Li, J.; Zhou, Q.; Cui, C.; Hosseinzadeh, P.; Mukherjee, A.; Nilges, M. J.; Wang, J.; Lu, Y. Defining the role of tyrosine and rational tuning of oxidase activity by genetic incorporation of unnatural tyrosine analogs. J. Am. Chem. Soc. 2015, 137, 4594-4597.

(35) Ravichandran, K. R.; Zong, A. B.; Taguchi, A. T.; Nocera, D. G.; Stubbe, J.; Tommos, C. Formal reduction potentials of difluorotyrosine and trifluorotyrosine protein residues: Defining the thermodynamics of multistep radical transfer. J. Am. Chem. Soc. 2017, 139, 2994-3004. 
(36) Jonsson, M.; Lind, J.; Eriksen, T. E.; Merenyi, G. Redox and acidity properties of 4-substituted aniline radical cations in water. J. Am. Chem. Soc. 1994, 116, $1423-$ 1427.

(37) Adamo, C.; Subra, R.; Di Matteo, A.; Barone, V. Structure and magnetic properties of benzyl, anilino, and phenoxyl radicals by density functional computations. J. Chem Phys. 1998, 109, 10244.

(38) Morris, J. L.; Reddington, S. C.; Murphy, D. M.; Jones, D. D.; Platts, J. A.; Tippmann, E. M. Aryl azide photochemistry in defined protein environments. Org. Lett. 2013, 15, 728-731.

(39) Pan, J.; Wenger, E. S.; Matthews, M. L.; Pollock, C. J.; Bhardwaj, M.; Kim, A. J.; Allen, B. D.; Grossman, R. B.; Krebs, C.; Bollinger, J. M., Jr. Evidence for modulation of oxygen rebound rate in control of outcome by iron(II)- and 2oxoglutarate-dependent oxygenases. J. Am. Chem. Soc. 2019, 141, 15153-15165.

(40) Kato, N.; Suzuki, H.; Takagi, H.; Uramoto, M.; Takahashi, S.; Osada, H. Gene disruption and biochemical characterization of verruculogen synthase of Aspergillus fumigatus. ChemBioChem 2011, 12, 711-714.

(41) Fornal, E.; Parfieniuk, E.; Czeczko, R.; Bilinska-Wielgus, N.; Frac, M. Fast and easy liquid chromatography-mass spectrometry method for evaluation of postharvest fruit safety by determination of mycotoxins: Fumitremorgin $\mathrm{C}$ and verruculogen. Postharvest Biol. Tec. 2017, 131, 46-54.

(42) Gray, H. B.; Winkler, J. R. Hole hopping through tyrosine/tryptophan chains protects proteins from oxidative damage. Proc. Natl. Acad. Sci. U.S.A. 2015, 112, 10920-10925.

(43) Baldwin, J.; Krebs, C.; Ley, B. A.; Edmondson, D. E.; Huynh, B. H.; Bollinger, J. M. Mechanism of rapid electron transfer during oxygen activation in the R2 subunit of Escherichia coli ribonucleotide reductase. 1. Evidence for a transient tryptophan radical. J. Am. Chem. Soc. 2000, 122, 12195-12206.

(44) Svistunenko, D. A.; Cooper, C. E. A new method of identifying the site of tyrosyl radicals in proteins. Biophys. J. 2004, 87, 582-595. 
(45) Oldemeyer, S.; Mittag, M.; Kottke, T. Time-resolved infrared and visible spectroscopy on cryptochrome aCRY: Basis for red light reception. Biophys. J. 2019, 117, 490-499.

(46) Ryle, M. J.; Liu, A.; Muthukumaran, R. B.; Ho, R. Y. N.; Koehntop, K. D.; McCracken, J.; Que, L., Jr.; Hausinger, R. P. O2- and a-ketoglutarate-dependent tyrosyl radical formation in TauD, an a-keto acid-dependent non-heme iron dioxygenase. Biochemistry, 2003, 42, 1854-1862.

(47) Yin, H.; Xu, L.; Porter, N. A. Free radical lipid peroxidation: mechanisms and analysis. Chem. Rev. 2011, 111, 5944-5972.

(48) Huang, X.; Groves, J. T. Beyond ferryl-mediated hydroxylation: 40 years of the rebound mechanism and C-H activation. J. Biol. Inorg. Chem. 2017, 22, 185-207.

(49) Huang, X.; Groves, J. T. Oxygen activation and radical transformations in heme proteins and metalloporphyrins. Chem. Rev. 2018, 118, 2491-2553.

(50) Tallman, K. A.; Pratt, D. A.; Porter, N. A. Kinetic products of linoleate peroxidation: rapid $\beta$-fragmentation of nonconjugated peroxyls. J. Am. Chem. Soc. 2001, 123, 11827-1182.

(51) Mahoney, L. R. Antioxidants. Angew. Chem. Int. Ed. 1969, 8, 547-555. 


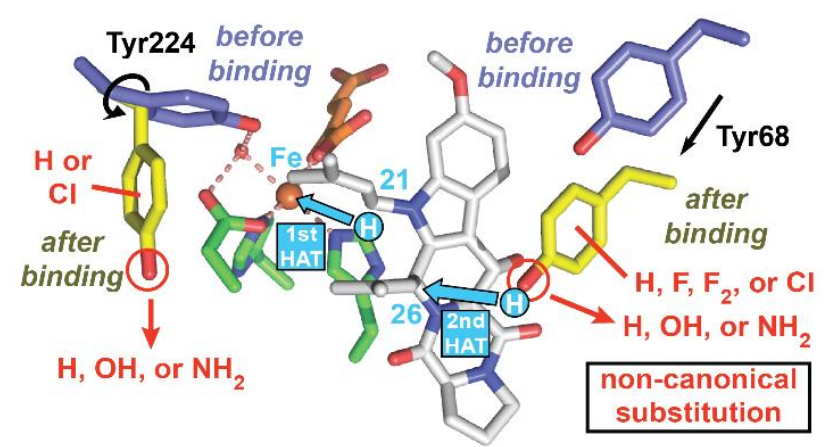

For Table of Contents Only 